ACCEPTED TO THE Astrophysical JOURnAL

Preprint typeset using LTEX style emulateapj v. 08/22/09

\title{
AN INCREASING STELLAR BARYON FRACTION IN BRIGHT GALAXIES AT HIGH REDSHIFT
}

\author{
Steven L. Finkelstein ${ }^{1, \mathrm{~A}}$, Mimi Song ${ }^{1}$, Peter Behroozi ${ }^{2}, \mathrm{R}_{\text {AChel S. Somerville }}^{3}$, Casey Papovich ${ }^{4}$, Miloš $^{2}$ \\ Milos aVluević ${ }^{1}$, Avishai Dekel ${ }^{5}$, Desika Narayanan ${ }^{6}$, Matthew L. N. Ashby ${ }^{7}$, Asantha Cooray ${ }^{8}$, Giovanni G. Fazio ${ }^{7}$, \\ Henry C. Ferguson ${ }^{2}$, Anton M. Koekemoer ${ }^{2}$, Brett SAlmon ${ }^{4}$, \& S. P. Willner ${ }^{7}$ \\ Accepted to the Astrophysical Journal
}

\begin{abstract}
Recent observations have shown that the characteristic luminosity of the rest-frame ultraviolet (UV) luminosity function does not significantly evolve at $4<z<7$ and is approximately $M_{\mathrm{UV}}^{*} \sim-21$. We investigate this apparent non-evolution by examining a sample of 178 bright, $M_{\mathrm{UV}}<-21$ galaxies at $z=4$ to 7 , analyzing their stellar populations and host halo masses. Including deep Spitzer/IRAC imaging to constrain the rest-frame optical light, we find that $M_{\mathrm{UV}}^{*}$ galaxies at $z=4-7$ have similar stellar masses of $\log \left(M / M_{\odot}\right)=9.6-9.9$ and are thus relatively massive for these high redshifts. However, bright galaxies at $z=4-7$ are less massive and have younger inferred ages than similarly bright galaxies at $z=2-3$, even though the two populations have similar star formation rates and levels of dust attenuation for a fixed dust-attenuation curve. Matching the abundances of these bright $z=4-7$ galaxies to halo mass functions from the Bolshoi $\Lambda \mathrm{CDM}$ simulation implies that the typical halo masses in $\sim M_{\mathrm{UV}}^{*}$ galaxies decrease from $\log \left(M_{\mathrm{h}} / M_{\odot}\right)=11.9$ at $z=4$ to $\log \left(M_{\mathrm{h}} / M_{\odot}\right)=11.4$ at $z=7$. Thus, although we are studying galaxies at a similar mass across multiple redshifts, these galaxies live in lower mass halos at higher redshift. The stellar baryon fraction in units of the cosmic mean $\Omega_{\mathrm{b}} / \Omega_{\mathrm{m}}$ rises from $5.1 \%$ at $z=4$ to $11.7 \%$ at $z=7$; this evolution is significant at the $\sim 3 \sigma$ level. This rise does not agree with simple expectations of how galaxies grow, and implies that some effect, perhaps a diminishing efficiency of feedback, is allowing a higher fraction of available baryons to be converted into stars at high redshifts.

Subject headings: early universe — galaxies: evolution — galaxies: formation — galaxies: high-redshift — ultraviolet: galaxies
\end{abstract}

\section{INTRODUCTION}

Tracing the buildup of stellar mass from the epoch of the first galaxies through the present can be used to constrain models of galaxy formation. Understanding what physics governs this buildup is one of the key outstanding questions in galaxy evolution. There is a consensus that the cosmic star formation rate (SFR) density rises from the dawn of galaxies, peaks at redshifts $z \sim 2-3$, and then declines steeply at $z<2$ (e.g., Madau \& Dickinson 2014). The physical origin of this evolution in galaxy stellar mass growth is poorly understood, especially at high redshifts. While studies of galaxy evolution routinely quantify the stellar content of distant galaxies, it remains challenging to relate the stellar masses to the supply of gas fueling star formation. Theoretical works attempt to address these fundamental questions to a varying degree of success, but observational data have remained incomplete, particularly at the massive end, and they are dominated by systematic uncertainties unavoidable in the stitching together

${ }^{1}$ Department of Astronomy, The University of Texas at Austin, Austin, TX 78712

${ }^{2}$ Space Telescope Science Institute, Baltimore, MD 21218

${ }^{3}$ Department of Physics \& Astronomy, Rutgers University, 136 Frelinghuysen Road, Piscataway, NJ 08854

${ }^{4}$ George P. and Cynthia Woods Mitchell Institute for Fundamental Physics and Astronomy, Department of Physics and Astronomy, Texas A\&M University, College Station, TX 77843

${ }^{5}$ Racah Institute of Physics, The Hebrew University, Jerusalem 91904, Israel

${ }^{6}$ Department of Physics and Astronomy, Haverford College, Haverford, PA 19041

${ }^{7}$ Harvard-Smithsonian Center for Astrophysics, 60 Garden Street, Cambridge, Massachusetts 02138

${ }^{8}$ Center for Cosmology, Department of Physics and Astronomy, University of California, Irvine, CA 92697

a stevenf@astro.as.utexas.edu of datasets from different campaigns (e.g., Behroozi et al. 2013a).

The advances facilitated by the Wide Field Camera 3 (WFC3) on the Hubble Space Telescope (HST) over the past half-decade have led to the discovery of $>1000$ redshift $z>$ 6 galaxies (e.g., Finkelstein et al. 2010, 2012a b, 2013, 2015; Bouwens et al. 2010, 2012, 2014, 2015; Oesch et al. 2010, 2012, 2013, 2014; McLure et al. 2010, 2013; Wilkins et al. 2011; Schenker et al. 2013). Among the detailed analyses facilitated by these large samples is the measurement of the rest-frame ultraviolet luminosity function, which quantifies the relative abundances of galaxies over a wide dynamic range in luminosity. As the UV light probes recent star formation activity, the integral of the rest-frame UV luminosity function provides an estimate of the cosmic SFR density (e.g., Madau et al.1996; Bouwens et al. 2012; Madau \& Dickinson 2014; Finkelstein et al. 2015). The luminosity function is typically parameterized with the Schechter (1976) functional form that is a power law at low luminosities and declines exponentially at high luminosities. Its parameters are the characteristic luminosity $M_{\mathrm{UV}}^{*}$, the faint-end slope $\alpha$, and the normalization $\phi^{*}$. Previous studies typically found that these parameters evolved with redshift: the characteristic luminosity decreased with increasing redshift as the faint-end slope steepened (e.g., Bouwens et al. 2007, 2011a b; McLure et al. 2013). This "luminosity evolution" of the luminosity function was widely accepted as it fit the general trend observable in the evolution of the cosmic SFR density.

More recent work, however, has shown that the picture described above is incomplete. The first evidence came from Ono et al. (2012), Finkelstein et al. (2013), and Bowler et al. (2014), where a larger than expected number of bright galaxies turned up as $z=7$ surveys moved to wider fields. 


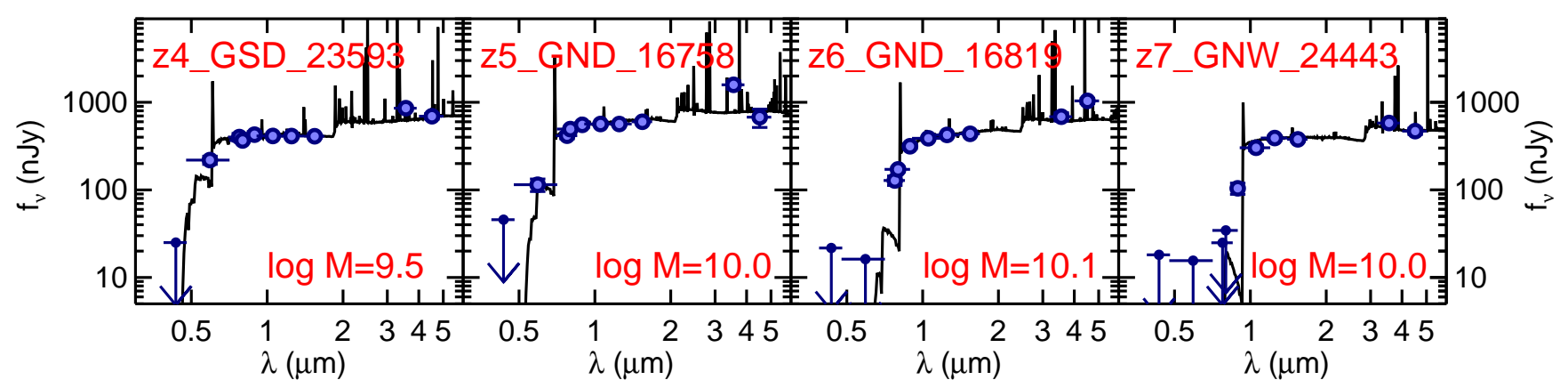

FIG. 1. - The spectral energy distribution of a representative galaxy in each of our redshift bins, shown as the blue circles. For filters where the signal-to-noise was $<2$, we show the $1 \sigma$ upper limits. The black curve shows the best-fit stellar population model, and we list the ID and best-fit stellar mass for each galaxy.

Finkelstein et al. (2015) and Bouwens et al. (2015) have confirmed this excess, and recent studies have concluded it is not attributable to gravitational lensing (Mason et al. 2015; Barone-Nugent et al. 2015). Both Finkelstein et al. (2015) and Bouwens et al. (2015) computed the evolution of the restframe UV luminosity function at $z=4-8$, finding that contrary to the preceding results derived from smaller datasets, the characteristic luminosity $M_{\mathrm{UV}}^{*}$ was remarkably redshiftindependent between $z=4,5,6$, and 7 . The constancy broke down only at $z=8$ where the data were least constraining. Most of the evolution took place in the characteristic number density: it declined towards higher redshifts. Therefore, while galaxies in general became less common at higher redshiftsconsistent with the decline in the cosmic SFR density-bright galaxies remained relatively common in the distant universe.

Here, we seek to constrain the physical properties in distant UV-bright galaxies and attempt to understand how they maintained high levels of star formation. In $\$ 2$, we describe the bright galaxy sample that we have taken from Finkelstein et al. (2015) and discuss the additional constraints that can be placed on the stellar populations with the inclusion of Spitzer/IRAC photometry. In §3, we use cosmic abundance matching to estimate the halo masses and the stellar-to-halo mass ratios for these galaxies. In $\$ 4$, we discuss the evolution of the stellar baryon fraction with redshift, and in $\$ 5$, we present our conclusions. We assume the WMAP7 $\Lambda \mathrm{CDM}$ cosmological model (Komatsu et al. 2011) throughout, with $H_{0}=70.2 \mathrm{~km} \mathrm{~s}^{-1} \mathrm{Mpc}^{-1}, \Omega_{\mathrm{m}}=0.275$, and $\Omega_{\Lambda}=0.725$. All magnitudes given are in the AB system (Oke \& Gunn 1983).

\section{STELLAR POPULATIONS IN UV-BRIGHT GALAXIES}

Here we wish to constrain the physical processes that regulate the abundance of bright galaxies in the distant universe. These luminous systems are observed only a short time after the Big Bang and trace prominent density peaks at their epoch. We further investigate sources with $M_{1500}<-21$. This magnitude is approximately the value of $M_{\mathrm{IV}}^{*}$ at these redshifts (Bouwens et al. 2015; Finkelstein et al. 2015), though the exact value of $M_{\mathrm{UV}}^{*}$ does get progressively more uncertain with increasing redshift $( \pm 0.09$ at $z=4$ to \pm 0.4 at $z=7$; Finkelstein et al. 2015). Using ground-based data, Bowler et al. (2015) have found evidence that $M_{\mathrm{UV}}^{*}$ may be fainter than -21 at $z=7$, though only at the $2 \sigma$ level, and thus not significantly discrepant with the measurements of Bouwens et al. (2015) and Finkelstein et al. (2015). Because this study is concerned with the physics driving the apparently high star-formation rates in distant bright galaxies, the exact luminosity we choose is not critical. In order to explore relatively bright galaxies, we choose the approximate value of
$M_{\mathrm{UV}}^{*}$ at these redshifts as our threshold.

We use the sample of $\sim 7500$ galaxies at $4 \lesssim z \lesssim 8$ from Finkelstein et al. (2015) and refer the reader to that paper for details on the photometry, photometric redshift sample selection, and derivation of UV absolute magnitude at $1500 \AA$ $\left(M_{\mathrm{UV}}\right)$. From their full catalog, here we analyze the 150,75 , 28,18 , and 3 galaxies with $M_{\mathrm{UV}}<-21$ at $z=4,5,6,7$, and 8 , respectively, which come from the CANDELS GOODSSouth and North fields (with a total area of $\sim 280 \mathrm{arcmin}^{2}$ ). The redshift bins are bounded by $\Delta z= \pm 0.5$ from the central redshift.

\subsection{Inclusion of Spitzer/IRAC Photometry}

To learn more about these intriguing systems, we turn to stellar population modeling. We use the HST photometry from Finkelstein et al. (2015) that includes ACS and WFC3 PSF-matched total fluxes in the wavelength range $0.4-1.8$ $\mu \mathrm{m}$ (see Koekemoer et al. 2011, for details on the imaging). This catalog also includes photometry from the Spitzer Space Telescope Infrared Array Camera (IRAC; Fazio et al. 2004) imaging of our fields. The IRAC imaging probes the restframe optical at $z>4$ and thus provides significant constraining power on the stellar masses of our galaxies. It also gives a more accurate handle on the ages and dust attenuations by reducing the degeneracy between the two parameters. The details of the long-wavelength photometry are presented in Finkelstein et al. (2015); here, we review them only briefly. The mosaics were obtained by coadding all the available data in these fields: the GOODS (Dickinson et al., in prep), Spitzer Extended Deep Survey (SEDS; Ashby et al. 2013), and Spitzer-CANDELS (S-CANDELS; Ashby et al. 2015) wide-field programs, as well as the deep pointings from Spitzer program 70145 (the IRAC Ultra-Deep Field of Labbé et al. 2013) over the Hubble Ultra Deep Field and its parallels, and program 70204 (PI Fazio) which observed a region in the GOODS-S field to $100 \mathrm{hr}$ depth. The final mosaics have a depth of $\gtrsim 50 \mathrm{hr}$ over both CANDELS GOODS fields and $>100 \mathrm{hr}$ over the HUDF main field (Ashby et al. 2015).

The TPHOT software (Merlin et al. 2015), an updated version of TFIT (Laidler et al. 2007), was used to measure photometry in the Spitzer/IRAC imaging. This software models the low-resolution IRAC images by convolving the $H S T / W F C 3 H$-band image with an empirically derived IRAC PSF, simultaneously fitting all IRAC sources. This provides robust photometry even for moderately blended sources. The full description of our TPHOT IRAC photometry catalog is presented in Song et al. (2015).

All high-redshift galaxies were visually inspected in the TPHOT residual maps. If an object was on or near a strong 
residual, reliable IRAC photometry was not possible, affecting $20-30 \%$ of the galaxies in our bright galaxy sample. To obtain the most robust stellar mass measurements, in our subsequent analysis we do not include these affected galaxies. Over $90 \%$ of the remaining galaxies in our sample had a 3.6 $\mu \mathrm{m}$ or $4.5 \mu \mathrm{m}$ detection of at least $3 \sigma$ significance, with a magnitude range at $z \geq 6$ of $23.5 \leq m_{3.6} \leq 25.5$. This is expected, as galaxies with $M_{1500}<-21$ should be massive enough at all redshifts to yield an IRAC detection absent crowding. In fact, when comparing median stellar masses derived excluding and including galaxies without IRAC constraints, the median stellar mass of galaxies with IRAC constraints is at most $\sim 0.1$ dex lower than that of the whole sample. This is because galaxies with true mid-infrared fluxes well below the IRAC detection limit can have poorly constrained SEDs. In contrast, our results show that the typical UV bright $z>6$ galaxy has a lower mass-to-light ratio than other possible solutions, thus the IRAC detection prior does not drive us to higher $\mathrm{M} / \mathrm{L}$ models.

The IRAC photometry used here is the same as by Finkelstein et al. (2015) who used the IRAC and additional HST/ACS F814W photometry to re-measure the photometric redshifts, removing 14,14 , and 1 galaxies from their $z=4$, 5 , and 6 samples, respectively. The galaxy sample we consider here comes from the cleaned sample of Finkelstein et al. (2015), thus these presumed lower redshift interlopers have already been removed. In this work, we have performed an additional iteration of visual inspection of the IRAC imaging, which results in a few more galaxies having preferred lower redshift solutions. From our sample we remove seven such additional galaxies which now have preferred lower redshift solutions (two from our $z=4$ sample, and five from our $z=5$ sample). We also remove five additional sources from our $z=4$ sample which have $2.7<z_{\text {phot }}<3.5$, as we do not wish to bias our $z=4$ sample stellar mass measurements. After excluding these objects, galaxies in the remaining sample all have photometric redshifts within $z_{\text {sample }}-0.5<z_{\text {phot }}<$ $z_{\text {sample }}+0.7$, thus we consider the effects of potential lowredshift interlopers to be minimal. The inclusion of the very small number of sources at $z_{\text {phot }}=z_{\text {sample }}+0.5-0.7$ (eight at $z=4$, six at $z=5$ and 1 at $z=7$; see tables in Appendix) make no difference to the median stellar mass discussed below. See Finkelstein et al. (2015) for a quantitative description of the potential contamination.

\subsection{Spectral Energy Distribution Fitting}

The technique we used to fit stellar population models to photometry was similar to the one we employed before (Finkelstein et al. 2010, 2012ba, 2013, 2015). We used the updated (2007) stellar population synthesis models of Bruzual \& Charlot (2003) to generate a grid of model spectra $^{1}$. We varied the stellar mass (defined as the total gas mass converted into stars), the stellar population metallicity, the time since the onset of star formation (henceforth, the

1 The 2007 update to the stellar population models of Bruzual \& Charlot (2003) may overestimate the contribution of thermally pulsating asymptotic giant branch (TP-AGB) stars. However, these stars typically begin to dominate the emission at population ages $\gtrsim 1 \mathrm{Gyr}$ and rest frame wavelengths $>1 \mu \mathrm{m}$. Our longest wavelength filter $(4.5 \mu \mathrm{m})$ at our lowest redshift $(z=$ 4) probes only $0.9 \mu \mathrm{m}$, and all other filter/redshift combinations probe bluer rest-frame wavelengths. The TP-AGB contribution may impact the SED in post-starburst galaxies at wavelengths as low as $0.5 \mu \mathrm{m}$ (Kriek et al. 2010). However, our galaxies are highly star-forming, with inferred population ages are $\ll 1$ Gyr. Thus, our choice to use the updated models likely has no effect on our results. age), and the star formation history (SFH). We assumed the Salpeter $^{2}$ initial mass function (IMF). Allowed metallicities spanned $(0.02-1) Z_{\odot}$ and ages spanned 1 Myr to the age of the universe at the source redshift. We allowed several different SFH scenarios, including a single burst, continuous star formation, and both the exponentially decaying and rising (so-called "tau" and "inverted-tau") models. We included nebular emission lines using the prescription of Salmon et al. (2015), which takes the line ratios from Inoue (2011), assuming that the gas has the same metallicity as the stars and that all the ionizing photons emitted by the model stellar population are reprocessed in the galaxy and their escape is negligible. To the rest frame spectra we added dust attenuation using the starburst attenuation curve of Calzetti et al. (2000) in the range of $0 \leq E(B-V) \leq 0.8\left(0 \leq A_{V} \leq 3.2 \mathrm{mag}\right)$. Then we redshifted the models to $0<z<11$ and added intergalactic medium (IGM) attenuation (Madau 1995). The resulting model spectra were integrated through our HST and Spitzer filter bandpasses to derive synthetic photometry for comparison with our observations.

We emphasize that our model parameterization assumes that the SFH of each object follows one of the simple scenarios (i.e., single burst, continuous, tau, or inverted-tau), not a superposition of such scenarios. This may seem like an oversimplification, but evidence is mounting that the SFHs in distant galaxies vary smoothly with time (e.g. Papovich et al. 2011; Finlator et al. 2011; Reddy et al. 2012b; Salmon et al. 2015). Therefore, the simple scenarios may in fact be rather good approximations, in particular when deriving the stellar mass (e.g., Lee et al. 2010). Therefore, the SFHs and thus the physical properties of the bright galaxies that we consider here should not be strongly affected by burstiness (e.g., Jaacks et al. 2012).

The best-fit model was found via $\chi^{2}$ minimization. We included an extra systematic error of $5 \%$ of the object flux in each band to crudely account for the residual uncertainties in the zero point correction and PSF matching process. The uncertainties in the best-fit parameters were derived via Monte Carlo simulations, perturbing the observed flux of each object in each filter with a number drawn from a Gaussian distribution with a standard deviation equal to the flux uncertainty in the filter. Taking the source redshift to be statistically uncorrelated with other spectral energy distribution (SED) fitting parameters, in each Monte-Carlo realization we drew the redshift from the photometric redshift statistical likelihood function of the given object. To prevent low-redshift solutions from biasing the physical parameters, we limited the random redshift to be within $\Delta z= \pm 1$ of the best-fit photometric redshift. This treatment of the source redshift effectively folded the uncertainty in redshift into the uncertainty in the physical parameters (most notably, the stellar mass and $M_{1500}$; Finkelstein et al. 2012a). For each galaxy, $10^{3}$ Monte Carlo realizations were generated and this provided a sample of as many values for each model parameter. SFRs for the best-fit model and for each Monte Carlo realization, were derived by converting the dust-corrected value of $M_{1500}$ to a SFR via the relation of Kennicutt (1998).

For our subsequent analysis, we discarded realizations with poor best-fit models with $\chi^{2}>20$ to ensure robustness of the derived properties. This removed a relatively small number of galaxies $(16,9,1,0$ and 1 at $z=4,5,6,7$ and 8 , respectively).

\footnotetext{
${ }^{2}$ To convert our results to those obtained from a Chabrier IMF, one should divide the stellar masses and SFRs by a factor of $\sim 1.7$.
} 


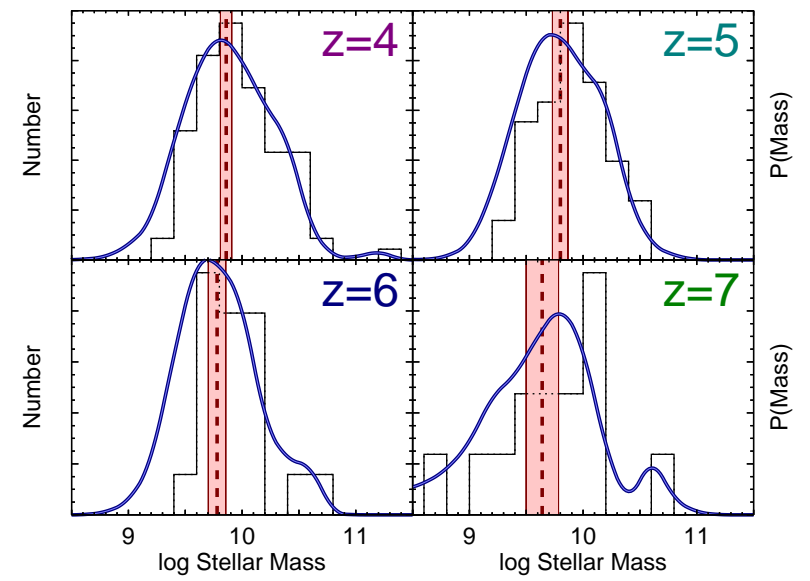

FIG. 2.- Joint probability distributions for the stellar masses of bright galaxies $\left(M_{1500}<-21\right)$ in our $z=4,5,6$ and 7 galaxy samples (blue line). The histograms are of the best-fit values, and the red dashed line and red shaded regions denote the median and $1 \sigma$ uncertainty on the median, respectively, of the joint PDFs. Little significant evolution in the median stellar mass is seen over this redshift range.

The final sample contains $94,46,19,14$, and 1 galaxy at $z=$ $4,5,6,7$, and 8 , respectively. As there is only one galaxy at $z=8$ that satisfies these criteria, we focus on $4<z<7$ for the remainder of this paper. Figure 1 shows the SED fit for a typical galaxy in each of our redshift bins.

\subsection{Physical Properties}

The results of SED fitting are summarized in Table 1 To derive the median stellar population properties, rather than stacking the images or fluxes of the galaxies, we stacked samples of Monte Carlo realizations in the model parameter space. In each redshift bin, the stacked sample allowed us to quantify the multivariate distribution of galaxy properties. The joint probability distribution functions for stellar mass marginalized over other parameters are shown in Figure 2. The median of a parameter such as the stellar mass is taken to be the median value in the bin. The $1 \sigma$ confidence interval on the median was calculated via $10^{3}$ bootstrap simulations where we rederived the median from a randomly drawn (with replacement) sample of the galaxies in each redshift bin.

As shown in Table 1, the median stellar population parameters do not evolve significantly with redshift. Broadly speaking, $M_{\mathrm{UV}}<-21$ galaxies at $z=4-7$ are moderately massive $\left(\log \left[M_{*} / M_{\odot}\right] \approx 9.6-9.9\right)$, somewhat young $(<100 \mathrm{Myr})$, and have non-negligible dust attenuation $(E(B-V)=0.07-0.13$ corresponding to $\left.A_{V} \approx 0.3-0.5 \mathrm{mag}\right)$ and high SFRs $(\sim 40-60$ $\left.M_{\odot} \mathrm{yr}^{-1}\right)$.

Significant amounts of dust are likely produced in galaxies as early as $z \sim 7$, as Finkelstein et al. (2012b) and Bouwens et al. (2014) have previously noted that massive and/or UV-bright galaxies had similarly red UV continuum slopes at $z=4-7$, with a typical value of the UV spectral slope $\beta \sim-1.8$. Both studies concluded that this implied a similar amount of dust in bright/massive $z=4-7$ galaxies, independent of redshift. We confirm this result, finding $E(B-V) \sim 0.1$ in bright galaxies at $z=4-7$ (constrained at $68 \%$ confidence to be $>0$ and $\lesssim 0.15$ ). Therefore, although fainter/lowermass galaxies appear to be less dusty at higher redshifts (e.g., Finkelstein et al. 2012b; Bouwens et al. 2014), this is not true for the brightest galaxies. This can be confirmed with ALMA, and in fact ALMA has recently detected dust emission from normal galaxies out to $z \sim 5-7.5$ (e.g., Watson et al. 2015;
TABLE 1

Median Physical Properties of Galaxies with $M_{1500}<-21$

\begin{tabular}{cccccc}
\hline \hline Redshift & Number & $\log \left(M_{*} / M_{\odot}\right)$ & $\begin{array}{c}\text { Age } \\
(\mathrm{Myr})\end{array}$ & $E(B-V)$ & $\begin{array}{c}\text { SFR } \\
\left(M_{\odot} \mathrm{yr}^{-1}\right)\end{array}$ \\
\hline $\mathrm{z}=4$ & 94 & $9.86 \pm 0.04$ & $44 \pm 2$ & $0.13 \pm 0.01$ & $56 \pm 4$ \\
$\mathrm{Z}=5$ & 46 & $9.80 \pm 0.06$ & $35 \pm 2$ & $0.12 \pm 0.02$ & $52 \pm 10$ \\
$\mathrm{Z}=6$ & 19 & $9.78 \pm 0.07$ & $40 \pm 4$ & $0.07 \pm 0.02$ & $40 \pm 8$ \\
$\mathrm{z}=7$ & 14 & $9.64 \pm 0.13$ & $29 \pm 8$ & $0.09 \pm 0.02$ & $41 \pm 9$ \\
\hline
\end{tabular}

NOTE. - Median values of physical parameters from the joint probability distribution describing all galaxies in a given redshift bin that have a measurement in the IRAC $3.6 \mu \mathrm{m}$ channel and a best-fit model with $\chi^{2}<20$. The number of galaxies satisfying these criteria in each redshift bin is given in the second column. The statistical uncertainties on median values were derived via $10^{3}$ Monte-Carlo simulations in which the median was rederived from a randomly drawn sample (with replacement) of the galaxies in each redshift bin. The spread in values for individual galaxies is larger.

Capak et al. 2015).

If the amount of UV attenuation due to dust among bright galaxies had evolved from $z=7$ to 4 , then it could have led to our selecting a lower stellar mass at a given UV absolute magnitude (a similar effect could occur if the dust attenuation curve is redshift dependent). However, our results show that this is not the case, as not only does our inferred attenuation exhibit no evolution, but the median stellar mass also appears roughly constant over the redshift interval.

Our fiducial analysis assumed a dust attenuation curve from Calzetti et al. (2000) for consistency with previous results in the literature. However, a number of recent studies have found that a Small Magellanic Cloud (SMC)-like (Pei 1992) attenuation curve may be more appropriate for high-redshift galaxies (e.g., Reddy et al. 2012a; Tilvi et al.2013; Capak et al.2015). This dust attenuation curve has more attenuation for given values of $E(B-V)$, thus we explored how our results would change had we assumed this attenuation curve in our fiducial analysis. Even with this different attenuation curve, the amount of dust attenuation appears to be roughly constant in these bright galaxies, with slightly lower values of $E(B-$ $\mathrm{V})=0.06-0.08$. We find that our median stellar masses would change by at most 0.14 dex (consistent with results from Papovich et al. 2001 that the choice of attenuation curve does not significantly affect stellar mass measurements), resulting in a minimal change to our major conclusion below on the stellar baryon fraction (although the slope of the stellar baryon fraction evolution assuming SMC dust is less than our fiducial scenario, and thus evolution is detected at reduced significance due to a lower value and larger uncertainty on the median mass of the $\mathrm{z}=7$ sample in this scenario). We conclude that our assumption of a Calzetti et al. attenuation curve does not result in a strong bias in our results.

The youngish character of these galaxies is surprising. Although age is notoriously difficult to measure robustly, the addition of the IRAC photometry does help, in particular at $z=6$, where the ensemble is constrained to have a typical age $<50$ Myr (68\% C.L.). While evolved galaxies are not absent at these high redshifts (for example, z7_GNW_17001 has $\log \left(M_{*} / M_{\odot}\right)=10.7$ and an age of $\left.400 \mathrm{Myr}\right)$, they seem to be exceptions. This is in stark contrast with galaxies at $z \lesssim 3$, where those residing at the bright-end of the luminosity function tend to be more evolved.

Reddy et al. (2006) published a stellar population analysis of galaxies at redshifts $z \sim 1-3.5$. Using the results from their 
models that assume a constant SFH, the median galaxy with $M_{\mathrm{UV}}<-21$ (derived from the observed R-band magnitude and spectroscopic redshift) at $z \sim 2-3$ has $\log \left(M_{*} / M_{\odot}\right)=$ 10.2 , an age of $260 \mathrm{Myr}, E(B-V)=0.16$ and $\mathrm{SFR}=70$ $M_{\odot} \mathrm{yr}^{-1}$ (the results are similar when the sample is split into two redshift bins centered at $z=2$ and $z=3$ ). Thus, bright galaxies at the peak of cosmic star-formation activity have similar SFRs and dust attenuations as bright galaxies in the epoch of reionization, but the lower redshift galaxies are $\sim 2$ $2.5 \times$ more massive and have rest-UV/optical light dominated by stars $>5 \times$ older. Such a comparison with lower redshift makes sense if one is interested in the evolution of properties of similarly bright galaxies with redshift. It is also valid if we wish to explore the evolution of galaxies with $M_{\mathrm{UV}}<M_{\mathrm{UV}}^{*}$ with redshift, as $M_{\mathrm{IIV}}^{*}$ at $z \sim 2.3$ and $3(-20.7$ and -20.97 , respectively; Reddy \& Steidel 2009) are similar to what we find at $z \geq 4$.

A more interesting question is how UV-bright galaxies at $z \sim 2-3$ are related to UV-bright galaxies at $z \sim 6-8$. Specifically, in view of the hierarchical galaxy assembly, are the former galaxies descendants of the latter? Are the latter progenitors of the former? Moreover, galaxy merging complicates direct number-counting-based matching across redshifts. A few recent studies have tried comparing galaxies at different redshifts at the same cumulative number density (e.g., van Dokkum et al. 2010; Papovich et al. 2011; Leia et al. 2013). Behroozi et al. (2013a) showed that such a comparison is adequate for identifying the low- $z$ descendant population of a high- $z$ population (see also Jaacks et al.2015). This is because the majority of massive high- $z$ galaxies do not end up merging into substantially more massive systems. However, the converse is not true: reflecting hierarchal merging, the cumulative number density of the high- $z$ progenitor population of a low $-z$ population has a comparatively higher cumulative number density.

As shown in the following subsection, galaxies at $z=6$ and 7 have $n\left(M_{\mathrm{UV}}<-21\right)=3.6 \times 10^{-5} \mathrm{Mpc}^{-3}$ and $2.4 \times$ $10^{-5} \mathrm{Mpc}^{-3}$, respectively. Using the luminosity functions of Reddy \& Steidel (2009) at $1.9<z<2.7$ (the median redshift of the $z \sim 2-3$ comparison sample we quote here is $z=2.44$ ), we find $n\left(M_{\mathrm{UV}}<-21\right)=2.24 \times 10^{-4} \mathrm{Mpc}^{-3}$, unsurprisingly more abundant than our high-redshift sample. To match the cumulative number densities of our high-redshift samples, we need to select objects with $M_{\mathrm{UV}}<-20.0$ and $<-19.8$ for our $z=6$ and 7 sample, respectively. The $M_{\mathrm{UV}}<-21$ galaxies at $z \sim 2-3$ are thus plausible descendants of the high- $z$ galaxies at these fainter magnitudes.

Although above we only report stellar population results for bright galaxies, we performed SED fitting on the entire sample of Finkelstein et al. (2015), finding that $M_{\mathrm{UV}}=-20$ galaxies at $z=6$ have mean stellar masses of $\log \left(M_{*} / M_{\odot}\right)=8.7$ and mean ages of 34 Myr. At $z=7, M_{\mathrm{UV}}=-19.8$ galaxies have median stellar masses of $\log \left(M_{*} / M_{\odot}\right)=8.5$ and median ages of $28 \mathrm{Myr}$. We conclude that, compared to either similarly bright or similarly abundant galaxies at lower-redshift, UV-bright galaxies at $z>6$ are significantly younger and less massive, but have similar SFRs, as inferred from their similar UV luminosities, and exhibit relatively little evolution of dust attenuation at these luminosities.

We acknowledge that ages are notoriously difficult to constrain, as they are tied to the assumed star-formation histories (e.g., Papovich et al. 2001). In more realistic scenarios, the young stars that dominate the observed SED are mod- estly "outshining" the older generations, potentially biasing the measured age. However, the stellar masses (which we use for our primary result in the following sections) are robust to age variations (Lee et al. 2010), thus this potential bias does not affect our main results.

\section{HALO MASSES OF UV-BRIGHT GALAXIES}

\subsection{Halo Masses}

Given the relatively high stellar masses and SFRs seen in our sample of $z \geq 6$ galaxies, one may wonder if the processes governing gas cooling and the conversion of cold gas into stars differ from those at lower redshift. As a step toward answering this question, we compare the stellar masses $M_{*}$ of these systems to the baryon masses in halos $\left(\Omega_{\mathrm{b}} / \Omega_{\mathrm{m}}\right) M_{\mathrm{h}}$ computed assuming a cosmic-mean baryon fraction of $\Omega_{\mathrm{b}} / \Omega_{\mathrm{m}}=0.1669$ (Komatsu et al.2011). The clustering of these systems would have provided the most direct constraints on the halo mass, but the numbers and surface densities of these galaxies, particularly at $z \geq 7$, are not yet sufficient to permit a robust clustering analysis (though see Barone-Nugent et al. 2014). Instead, we use abundance matching to estimate the halo masses for our bright galaxies. We refer the reader to previous works for a full discussion of abundance matching (e.g., Moster et al. 2010; Behroozi et al. 2010). Here, we review the procedure only briefly.

Abundance matching assumes that the galaxy luminosity or stellar mass is a monotonic function of the halo mass. The most luminous galaxies are assumed to live in the most massive halos. This is certainly a plausible assumption among the luminous galaxies we study here, but perhaps not among the more stochastic dwarf galaxies. Starting with a cosmological simulation (in this case, the pure $\Lambda \mathrm{CDM}$ simulation Bolshoi), one selects simulation snapshots close in redshift to the target redshift of an observational survey. In these snapshots, one selects a simulation volume equal to the volume of the survey. One then identifies all virialized halos in the volume and rank-orders them by mass. After rank-ordering the observed galaxies by their luminosities, one places each galaxy in the simulated halo of the matching rank. This procedure provides a mapping of galaxy statistics onto host halo statistics.

Here, we use Schechter parameterization of the observed luminosity functions at each redshift from Finkelstein et al. (2015) to estimate the host halo masses for the bright $\left(M_{1500}<\right.$ -21) galaxies in our sample. Given the present stellar mass function uncertainties, luminosity-based matching is currently more robust than the stellar mass-based matching. In the future, the matching should be performed directly with the stellar mass as it should be more correlated than the UV luminosity with the host halo mass (e.g., Lee et al. 2009; Gerke et al. 2013).

For our analysis, we use the results from the Bolshoi cosmological simulations (Klypin et al. 2011), which has $2048^{3}$ particles in a $250(\mathrm{Mpc} / h)^{3}$ box. This translates to a halo mass resolution of $\log \left(M_{\mathrm{h}} / M_{\odot}\right)=10$. As we will find that our galaxies have halo masses $\gg 10^{10} \mathrm{M}_{\odot}$, the resolution of Bolshoi is more than sufficient for the present analysis. We use the halo mass functions derived in Behroozi et al. (2013b), which are a modification of the Tinker et al. (2008) mass functions, include subhalos, and are accurate at very high redshifts (see Appendix G of Behroozi et al. 2013b). We derived halo mass functions at our redshifts of interest by volumeaveraging the Bolshoi snapshot mass functions over the same redshift ranges as those defining our galaxy samples. 

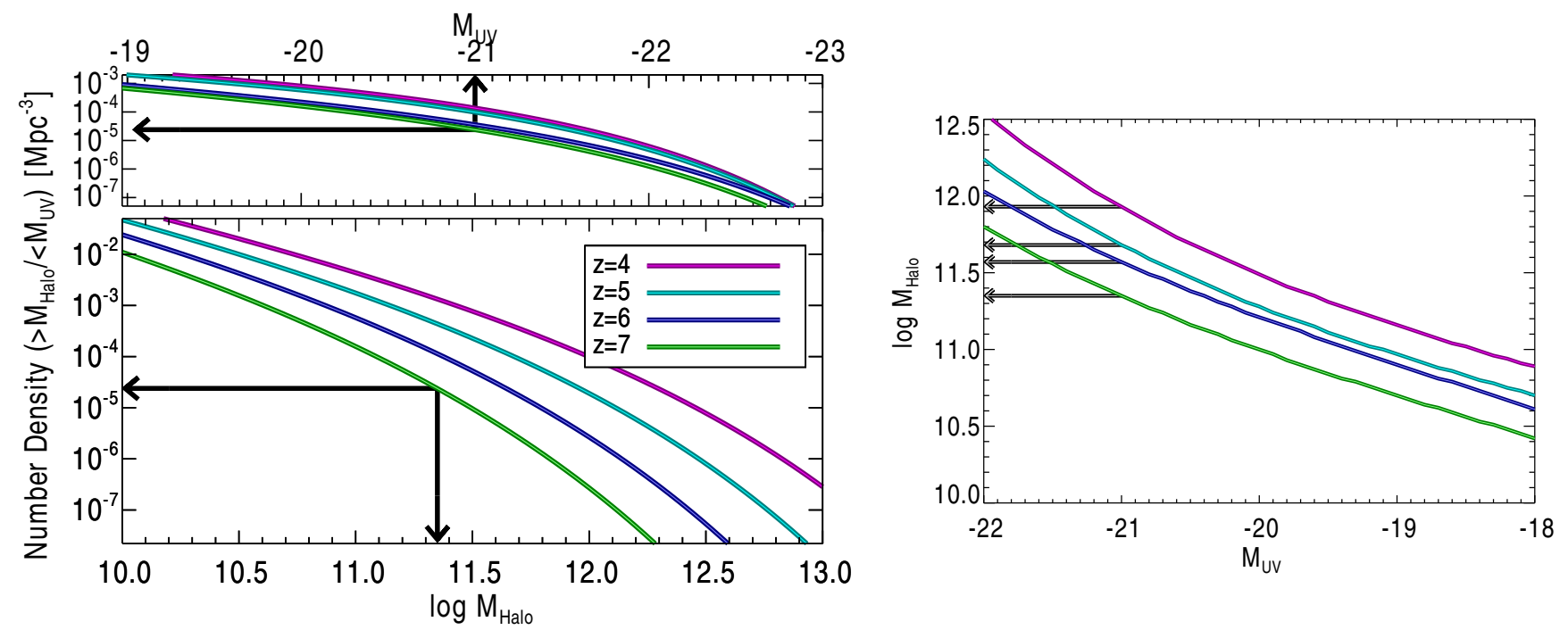

FIG. 3.- Top left: The cumulative luminosity function at $z=4,5,6$, and 7. Bottom Left: Cumulative halo mass functions at $z=4,5$, 6, and 7, derived by volume-averaging the Bolshoi snapshot mass functions over the same redshift ranges as those defining our galaxy samples. The arrows show our results from abundance matching at $z=7$, where galaxies with $M_{1500}<-21$, which have $n\left(M_{\mathrm{UV}}<-21\right)=2.5 \times 10^{-5} \mathrm{Mpc}^{3}$, have halo masses of $\log \left(M_{\mathrm{h}} / M_{\odot}\right)=11.35$. Right: Relation between observed UV absolute magnitude and abundance-matching-derived halo mass at our redshifts of interest. The arrows denote the halo masses at our magnitude of interest of $M_{\mathrm{UV}}=-21$.

The top-left panel of Figure 3 shows the cumulative luminosity functions at $z=4-7$ and the bottom-left panel shows the cumulative halo mass functions at the same redshifts. To infer the host halo masses for the bright galaxies with $M_{1500}<-21$, we first find the cumulative number density of galaxies at that magnitude. For example, for $z=7$, the number density is indicated with the horizontal arrow in the two left panels of Figure 3. The host halo masses can then be inferred by finding the halo mass above which the cumulative halo number density equals the cumulative galaxy density. We find $\log \left(M_{\mathrm{h}} / M_{\odot}\right)=11.93,11.68,11.57$, and 11.35 at $z=4,5,6$, and 7 , respectively. To estimate the uncertainties in the halo masses, we ran $10^{3}$ Monte Carlo simulations, in each drawing a luminosity function randomly from the MCMC sample generated during the luminosity function estimation, which account for both Poisson noise and uncertainties in the luminosity function completeness simulations (see Finkelstein et al. 2015). We find that the uncertainties in the halo mass are low, $\sim 0.01-0.03$ dex, reflecting relatively low uncertainties in the cumulative luminosity functions.

\subsubsection{Cosmic Variance}

To estimate how our measured abundances of bright galaxies are affected by cosmic variance, we used a suite of semianalytic models. These models, based on Somerville et al. (2008), were provided to the CANDELS team, and include a set of mock catalogs, one for each of the five CANDELS fields (with each individual mock catalog covering a volume somewhat larger than the observed volume). We tuned the dust attenuation in these SAMs to match the observed UV luminosity functions from Finkelstein et al. (2015). In each of our redshift bins, we used the SAMS to extract 64 independent volume elements comparable to one GOODS-sized field $\left(16^{\prime} \times 10^{\prime}\right)$ and 3192 independent volume elements comparable to a single WFC3 pointing $\left(2.1^{\prime} \times 2.1^{\prime}\right)$. In each redshift bin, the fractional uncertainty due to the combination of Poisson fluctuations and cosmic variance was derived as the standard deviation (computed over all of the realizations of a given field size) of the number of galaxies in a luminosity bin of $\Delta M_{\mathrm{UV}}=0.5 \mathrm{mag}$ centered at $M_{\mathrm{UV}}=-21$, divided by the mean number of galaxies in the bin.

The total fractional uncertainty due to Poisson fluctuations and cosmic variance was then derived by combining the variances from two GOODS-sized fields and five single WFC3 pointing-sized fields, as this was the area used in the luminosity function calculation by Finkelstein et al. (2015), who used GOODS-S, GOODS-N, and five individual fields (the HUDF, the two HUDF parallels, and the first two first-year Frontier Fields parallel fields). We find that the fractional uncertainties due to cosmic variance are $0.132,0.159,0.212$, and 0.327 at $z=4,5,6$, and 7 , respectively. We include these uncertainties in the Monte Carlo simulations discussed in the above paragraph, and find that the uncertainty in the derived halo mass increases by a factor of $\sim 2$ to 0.03 dex at $z=4$ and 0.06 dex at $z=7$ with the inclusion of the uncertainty due to cosmic variance. Our derived halo masses, and these total uncertainties, are listed in Table 2.

\subsection{Evolution of the Stellar-to-Halo Mass Ratio}

Comparing the halo masses estimated in the previous subsection to the median stellar masses estimated in $\$ 2.3$, we can calculate the ratio of the median stellar mass to halo mass (SMHM). We find $M_{*} / M_{\mathrm{h}}=0.009,0.013,0.016$, and 0.020 at $z=4,5,6$, and 7, respectively. Thus, at a constant UV luminosity, the stellar-to-halo mass ratio increases with increasing redshift. These results are listed in Table 2.

The cosmic baryon mass fraction is much higher than the $M_{*} / M_{\mathrm{h}}$ ratios we derive, at $\Omega_{\mathrm{b}} / \Omega_{\mathrm{m}}=0.1669$ Komatsu et al. 2011). In Figure 4 we show the evolution of the SMHM ratio in the units of $\Omega_{\mathrm{b}} / \Omega_{\mathrm{m}}$ as a function of redshift. We refer to this quantity as the stellar baryon fraction (SBF), as it measures the amount of baryons converted into stars compared to the cosmic allotment of baryons in the halo. We find that the SBF evolves from $0.117 \pm 0.043$ at $z=7$ to $0.051 \pm 0.006$ at $z=4$. This factor of $\sim 3$ evolution is significant, as fitting a linear function for $\operatorname{SBF}(z)$ yields the slope $d \mathrm{SBF} / d z=0.0239 \pm 0.0074$, with the trend detected at $3.2 \sigma$ significance. 


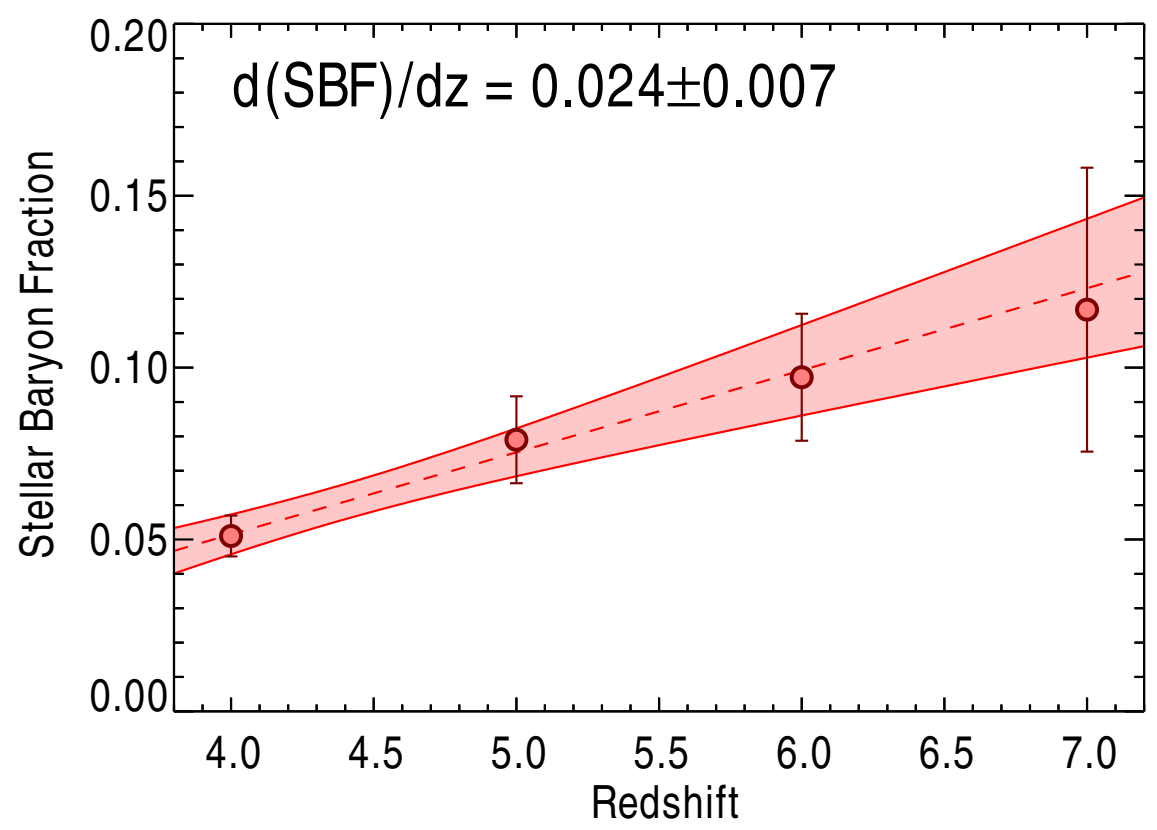

FIG. 4.- The stellar baryon fraction $(\mathrm{SBF})$ in bright $\left(M_{\mathrm{UV}}=-21\right)$ galaxies from $z=4$ to 7 . We define the $\mathrm{SBF}$ as the stellar to halo mass ratio in units of the cosmic baryon mass fraction $\Omega_{\mathrm{b}} / \Omega_{\mathrm{m}}$. We find that the SBF increases with increasing redshift, which may be responsible for the apparent lack of evolution in the characteristic magnitude $M_{\mathrm{UV}}^{*}$ observed over this redshift range.

The inferred significance of the trend depends on our assumed uncertainties in the stellar baryon fractions. We assumed an uncertainty in the halo mass and median stellar mass as reported in Table 2 . The quoted stellar mass uncertainties, which decrease from 0.14 dex at $z=7$ to 0.04 dex at $z=4$, are well below the uncertainty for an individual object, as here we are interested in the accuracy with which we can constrain the median stellar mass at different redshifts. However, these values are consistent with the typical uncertainty in the median stellar mass at $M_{\mathrm{UV}}=-21$ derived by Song et al. (2015), who fit the mass-to-light ratio over a wide dynamic range in UV luminosity, finding that it decreases from 0.1 dex at $z=7$ to 0.02 dex at $z=4$. If one used the median of individual galaxy mass uncertainties in each of our samples, which decreases from 0.19 dex at $z=7$ to 0.11 dex at $z=4$, the trend of increasing SBF with redshift is still apparent (albeit reduced in significance to $\sim 1.7 \sigma$ ).

Finally, we recall that our fiducial sample was selected to include galaxies with $M_{\mathrm{UV}}<-21$. Given the shape of the luminosity function, the median magnitude of this sample is very close to -21 (ranging from -21.2 to -21.3 ). To check if our sample selection biases the median masses, we examined how the evolution of the stellar baryon fraction changes if we use the median stellar mass of galaxies with luminosities $M_{\mathrm{UV}}=-21 \pm 0.25$. We find a comparable number of galaxies as in our fiducial sample, specifically 118, 76, 20, and 17 galaxies at $z=4,5,6$, and 7 , respectively. The median stellar mass is slightly lower than in our fiducial sample, $\log \left(M_{*} / M_{\odot}\right)=9.68,9.66,9.50$ and 9.52 and $z=4,5$, 6 and 7, respectively. The amplitude of the redshift derivative of the stellar baryon fraction is thus lower, $d \mathrm{SBF} / d z=$ $0.0123 \pm 0.0041$, yet the evolution is still significant at the $3.1 \sigma$ level. We conclude that there is significant evolution in the stellar baryon fraction in that it decreases with decreasing redshift, and that this evolution is stable against several def- initions of both the median stellar mass and the stellar mass uncertainty.

\subsubsection{Comparison to Previous Results}

Behroozi et al. (2013b) recently studied the evolution of the SMHM relation by modeling all available observational constraints, including luminosity functions, stellar mass functions, and SFRs, and exploring the galaxy evolution parameter space with an MCMC search. They found that the SMHM curve peaks at a roughly constant halo mass of $\log \left(M_{\mathrm{h}} / M_{\odot}\right)=$ 11.7 at $z=0-4$. Specifically, at $z=4$, they found a peak SBF at a halo mass of $\log \left(M_{\mathrm{h}} / M_{\odot}\right) \approx 12.0$, consistent with the typical halo mass we derive for our bright $z=4$ galaxies, $\log \left(M_{\mathrm{h}} / M_{\odot}\right)=11.9$. Then they went on to find that the peak of the SMHM relation at $z \geq 5$ occurs at a halo mass steadily decreasing with redshift, $\log \left(M_{\mathrm{h}} / M_{\odot}\right)=11.9,11.6$, and 11.4 at $z=5,6$, and 7 , respectively. This is very similar to what we find for the typical halo masses of $M_{\mathrm{UV}}^{*}$ galaxies, $\log \left(M_{\mathrm{h}} / M_{\odot}\right)=11.7,11.6$, and 11.4 at $z=5,6$, and 7 , respectively. The peak SMHM ratio measured by Behroozi et al. (2013b) is somewhat higher, $\sim 3.7 \%$ at $z=7$ (converting from a Chabrier to our Salpeter IMF) than the $2.0 \%$ we find here. However the two values are indistinguishable given the significant uncertainties in the high-redshift observables, particularly the stellar mass functions used by Behroozi et al. (2013b).

Although here we are specifically concerned with the halo masses of bright galaxies, in the right-hand panel of Figure 3 we also provide the abundance-matching-derived halo masses at all observed magnitudes in each of our redshift bins. Our derived halo mass of $\log \left(M_{\mathrm{h}} / M_{\odot}\right)=11.35$ at $z=7$ is consistent with a recent clustering-based measure of $\log \left(M_{\mathrm{h}} / M_{\odot}\right) \approx$ 11.2 from Barone-Nugent et al. (2014). However, their halo mass estimate was for galaxies with $M_{\mathrm{UV}}<-19.4$, a sample that has a lower average luminosity than our sample, and 
TABLE 2

Dark Matter Halo Properties of Bright Galaxies

\begin{tabular}{ccccc}
\hline \hline Redshift & $\begin{array}{c}\log n\left(M_{\mathrm{UV}}<-21\right) \\
\left(\mathrm{Mpc}^{-3}\right)\end{array}$ & $\begin{array}{c}\log M_{\mathrm{h}} \\
\left(M_{\odot}\right)\end{array}$ & $\begin{array}{c}\text { Median } \\
M_{*} / M_{\mathrm{h}}\end{array}$ & $\begin{array}{c}\text { Stellar Baryon } \\
\text { Fraction }\end{array}$ \\
\hline $\mathrm{z}=4$ & -3.86 & $11.93_{-0.03}^{+0.03}$ & $0.009 \pm 0.001$ & $0.051 \pm 0.006$ \\
$\mathrm{z}=5$ & -4.01 & $11.68_{-0.03}^{+0.04}$ & $0.013 \pm 0.002$ & $0.079 \pm 0.013$ \\
$\mathrm{z}=6$ & -4.45 & $11.57_{-0.03}^{+0.06}$ & $0.016 \pm 0.003$ & $0.097 \pm 0.019$ \\
$\mathrm{z}=7$ & -4.62 & $11.35_{-0.06}^{+0.09}$ & $0.020 \pm 0.007$ & $0.117 \pm 0.043$
\end{tabular}

Note. - The uncertainties in the halo mass are derived via Monte Carlo simulations and include the uncertainty in the number density of $M_{\mathrm{UV}}<-21$ galaxies, which reflects our fiducial luminosity function uncertainties as well as cosmic variance. The uncertainties in $M_{*} / M_{\mathrm{h}}$ and the stellar baryon fraction assume an uncertainty in the median stellar mass from Table 1.

thus a lower halo mass is expected, as shown in the right-hand panel of Figure 3. Our $z=4$ results are consistent with earlier clustering-based estimates by Lee et al. (2006), while at $z=$ 5 and $z=6$, our derived halo masses are somewhat higher than the clustering-based estimates from Lee et al. (2006) and Overzier et al. (2006), respectively, likely due to the fainter luminosities considered in those works $\left(M_{\mathrm{UV}}<-20-19.5\right.$, respectively). Although there are minor differences, it is encouraging that two independent methods, abundance matching and clustering, tentatively agree on the halo mass estimates at such high redshifts, though certainly the clusteringbased can be made more robust at $z>6$.

\subsubsection{UV Luminosity Scatter}

The halo mass estimates in $\S 3.1$ did not explicitly model the effect of the scatter in UV luminosity at a fixed halo mass. The estimates compared the number density in galaxies with $M_{\mathrm{UV}}<-21$ to the number density in halos with masses $>M_{\mathrm{h}}$ to derive the characteristic halo masses $M_{\mathrm{h}}$. The halo mass-luminosity relation has an intrinsic scatter that is usually treated as a Gaussian in the magnitude $M_{\mathrm{UV}}$ centered on some median (or mean) halo-mass-dependent magnitude. Because of this scatter, some halos with masses $>M_{\mathrm{h}}$ can can host galaxies with atypically low luminosities, $M_{\mathrm{UV}}>-21$, placing them outside of our luminosity cut. However, in $\$ 3.1$ the halos hosting these faint interlopers were being counted in the cumulative halo mass function and this could have biased the derived $M_{\mathrm{h}}$. If we had known which halos hosted faint interlopers (which we do not), we would have excluded them from the halo counting to derive a more accurate $M_{\mathrm{h}}$. Excluding a fraction of halos at every halo mass, the resulting characteristic halo mass $M_{\mathrm{h}}$ would have been lower than the scatter-blind estimate (Behroozi et al. 2010).

Here, we attempt to quantify this bias. We estimate the amplitude of the luminosity scatter from the relation between the UV luminosity and the stellar mass derived by Song et al. (2015). This is warranted because the stellar mass is expected to be more correlated with the halo mass than with UV luminosity. We find that at the mass of our sample $\log \left(M_{*} / M_{\odot}\right) \sim 9.8$, the scatter in the UV absolute magnitude is $\sim 0.3$ dex at all redshifts we consider.

To assess the impact of luminosity scatter on our abundance matching-determined halo masses, we carried out the iterative deconvolution described in Reddick et al. (2013) to get a handle on the true, underlying bivariate distribution of halo masses and observed UV luminosities that exhibits an intrinsic luminosity scatter at fixed halo mass. We performed abun- dance matching on the bivariate distribution properly applying the $M_{\mathrm{UV}}<-21$ cut to find halo masses $\log \left(M_{\mathrm{h}} / M_{\odot}\right)=$ $11.65,11.44,11.36$, and 11.13 at $z=4,5,6$, and 7 , respectively. These values are $\sim 0.2-0.25$ dex lower than the values obtained by scatter-blind abundance matching in $\S 3.1$ Therefore we expect that the true host halo masses of our $M_{\mathrm{UV}}<-21$ galaxies are slightly lower than those reported in Table 2. If the luminosity scatter had been $0.5 \mathrm{dex}$, our halo masses would be overestimated by $0.7-0.9$ dex.

Since halo masses at all redshifts are corrected by a similar logarithmic increment, the effect of luminosity scatter does not affect our detection of an evolving stellar baryon fraction. But for higher luminosity scatter which could characterize a different regime in which galaxy formation is more stochastic, this effect could be more significant.

\subsubsection{Systematic Uncertainties in Stellar Mass}

Our derivation of the stellar masses required a variety of assumptions that could have potentially biased our results. Could any of the biases explain away our conclusion that UVbright galaxies from $z=4$ to 7 have similar stellar masses but are found in progressively lower mass halos toward higher redshifts? One strong assumption we made is that of the Salpeter IMF. If the high-mass slope of the IMF evolved with redshift, this certainly could have biased the conclusion. Other assumptions involve the parameterization of SFHs and the treatment of metallicities and dust attenuations. As discussed earlier, the UV-continuum slopes in UV-bright galaxies appear to be roughly constant across this redshift range, and so dust abundances will only affect our results if the typical dust-law changes as a function of redshift. Direct measurement of the metallicities is beyond our current capabilities at these high redshifts (though see Finkelstein et al. 2013), but a plausible metallicity variation produces only a minor change in colors. The SFH parametrization is potentially more troubling, though recent results indicate that at least on average, galaxy-scale SFRs are smooth functions of time (e.g., Salmon et al. 2015).

A basic test of any bias in the mass measurements is to compare the shapes of the SEDs of our galaxies. Figure 5 shows a median flux stack of galaxies in each of our redshift bins versus the rest-frame wavelength, scaled vertically to a common redshift $z=6$. The shapes of the SEDs are remarkably similar, especially in the rest-frame UV, where they appear identical. Modest differences are visible in the rest-frame optical, likely due to the lower signal-to-noise of Spitzer/IRAC data, as well as the strong nebular [O III] and $\mathrm{H} \alpha$ lines which redshift through the bandpasses. Given the highly similar SED shapes, it is unlikely that an unaccounted for systematic effects strongly bias our results. Rather, we appear to be studying a very similar type of galaxy at each redshift; this type of galaxy lives in lower mass halos at higher redshift. This conclusion is confirmed by stellar population model fits to the stacks, which yield stellar masses consistent within $\sim 0.3$ dex of the median stellar masses in Table $1\left(\log \left[M_{*} / M_{\odot}\right]=9.8\right.$, 10.0, 9.9 and 9.9 at $z=4,5,6$, and 7 , respectively).

\subsubsection{Dusty Star-Forming Galaxies}

Our sample is selected on the basis of UV luminosity and therefore it is prudent to examine what effect that may have on our results. In particular, a rest-frame UV selection may miss extremely dusty galaxies which have their UV light attenuated below our detection sensitivity. Such systems, re- 


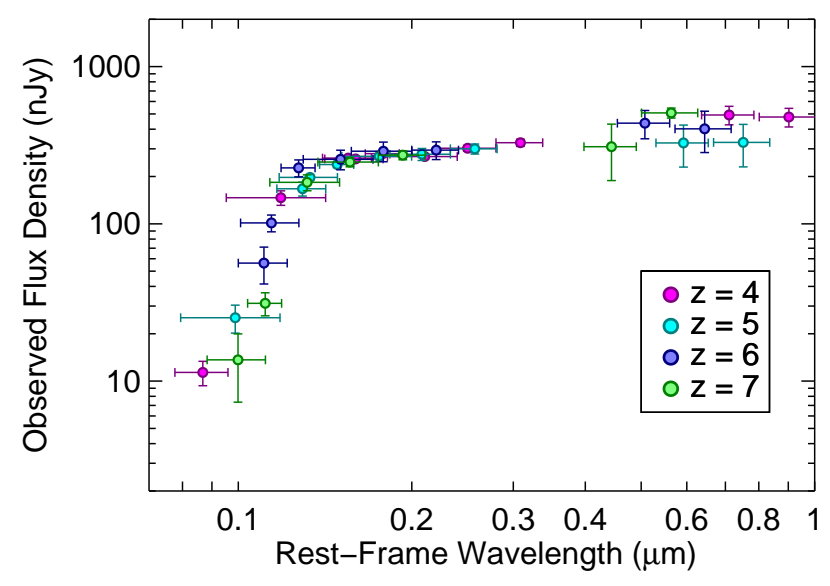

FIG. 5.- The median flux-stacked SEDs of our samples of bright galaxies at each redshift. The spectral shapes of these SEDs are remarkably similar, with minor differences appearing at longer wavelengths due to the presence of nebular emission lines, as well as the generally lower signal-to-noise of the long-wavelength data. The near identical nature of these SEDs confirms our conclusion that bright galaxies from $z=4$ to 7 are physically very similar, and that these $\log \left(M_{*} / M_{\odot}\right)=9.6-9.9$ galaxies on average inhabit lower mass halos at higher redshifts.

ferred to as sub-millimeter galaxies (SMGs; after their selection wavelength), have been well studied at moderate redshifts. These galaxies are typically very massive $\left(\log \mathrm{M} / \mathrm{M}_{\odot}\right.$ $>11$; Casey et al. 2014). If we were missing a large population of these galaxies, it would bias our derived halo masses to be too high (as we would be placing our observed galaxies in the most massive halos in our volume, which would truly be occupied by these dusty galaxies). If the abundance of SMGs evolves with redshift at $z>4$, then not accounting for these systems would bias our halo masses differently at different redshifts, biasing our inferred evolution of the stellar baryon fraction.

To explore the potential bias introduced by our UV-only selection, we compare the space density of SMGs at $z=4$ to our UV luminosity function. The space density of SMGs at high redshift is very uncertain for a variety of reasons, including the relatively shallow depths of sub-millimeter surveys, and the difficulty of obtaining redshifts for such systems. We start at $z=2$, where Chapman et al. (2005) found a space density for SMGs of $5 \times 10^{-5} \mathrm{Mpc}^{-3}$. A more complete survey for SMGs at multiple wavelengths was performed by Casey et al. (2013), who found a SMG SFR density $\sim 2 \times$ higher (see Fig. 23 of Casey et al. 2014), implying a space density of $z=2$ SMGs of $10^{-4} \mathrm{Mpc}^{-3}$. To estimate the evolution in this quantity to $z=4$, we use the redshift evolution shown by Casey et al. (2014), which shows that the SFR density from SMGs is an order of magnitude lower at $z \sim 4$ than at $z \sim 2$. Therefore we adopt $10^{-5} \mathrm{Mpc}^{-3}$ as a fiducial space density for $z=4$ SMGs.

We simulated the absence of SMGs in our sample by adding this abundance to our observed cumulative luminosity functions, and repeating the abundance matching analysis. The absence of such a population of SMGs results in a bias of the $z=4$ halo mass for our galaxies of interest of only $0.02 \mathrm{dex}$ (11.91 versus our fiducial result of 11.93). This is within the $68 \%$ confidence range on our fiducial halo mass, therefore not significant. If the abundance of SMGs stays constant to $z=$ 7, the declining UV luminosity function results in a slightly larger overestimate of the halo mass of 0.06 dex at $z=7$. Were this the case, the observed evolution in the stellar baryon fraction would be even stronger than we observe. However, such a high abundance of dusty galaxies at $z=7$ is highly unlikely. We therefore conclude that our selection does not affect our main conclusions in this study.

\section{DISCUSSION}

To understand the physical effects responsible for our observed trend of an increasing SBF with increasing redshift, here we consider a variety of possible mechanisms, with the caveat that our observations cannot uniquely distinguish between these scenarios. First, galactic gas at higher redshifts has higher surface densities $\Sigma_{\mathrm{g}} \propto f_{\text {gas }} M_{\mathrm{h}}^{1 / 3}(1+z)^{2}$, where $f_{\text {gas }}$ is the gas fraction in the cooled, virialized phase. The typical free-fall time to which the gas-to-stars conversion rate is proportional (albeit with a small coefficient-dimensionless SFR-see, e.g., Krumholz et al. 2012) is $t_{\mathrm{FF}} \propto(1+z)^{-3 / 2}$. However, masses of the most massive progenitors (MMP) of our galaxies' host halos are $M_{\mathrm{h}, \mathrm{MMP}}\left(z^{\prime}\right)=M_{\mathrm{h}}(z) e^{-\alpha\left(z^{\prime}-z\right)}$ with $\alpha \approx 1$, where $z^{\prime}>z$ is the progenitor redshift (Neistein et al. 2006; Fakhouri et al. 2010). Therefore the mass-doubling growth time is $t_{\text {grow }}(z) \sim\left[(1+z) H(z) d \ln M_{\mathrm{h}, \mathrm{MMP}} / d z\right]^{-1} \propto(1+$ $z)^{-5 / 2}$. This means that the ratio of the free-fall time to the growth time increases with increasing redshift as $t_{\mathrm{FF}} / t_{\text {grow }} \propto$ $1+z$, suggesting that if the minor progenitors are inefficient star formers so that they do not contribute substantial stellar mass to the main branch, it should be progressively more difficult at high redshifts to convert the gas acquired through growth into stars (Bouché et al. 2010; Krumholz \& Dekel 2012; Dekel et al. 2013). Second, the gas-phase metallicity and associated dust abundance appear to decrease with increasing redshift and decreasing mass (e.g., Finkelstein et al. 2012b; Bouwens et al.2014). Because metals and dust are the principal gas-cooling and UV-shielding agents, the observed trend of decreasing dust with increasing redshift could have a dramatic effect on the abundance of the cold $(T \lesssim 1000 \mathrm{~K})$ gas in which star formation seems to exclusively happen (Krumholz \& Dekel 2012; Krumholz et al. 2012). The drop in cold gas abundance toward higher redshifts would imply lower SFRs.

All this suggests that from the supply-versus-consumption side alone, we expect an opposite trend from the one measured, a trend in which the stellar baryon fraction decreases with increasing redshift. How should we then interpret the stellar baryon fractions that increase with increasing redshift? We can only speculate. Our observations imply that cold gas is less readily available for star-formation at lower redshifts. How might this come about? One scenario involves the transition of gas from the warm to cold phase, which occurs at a density $\sim 0.1-1 \mathrm{~cm}^{-3}$ (e.g., Wolfire et al. 1995, 2003). As the gas density decreases towards lower redshift, a progressively smaller mass fraction of the neutral gas phase is cold. Additionally, the nature of gas collapse in the disk is critical, as the galaxy-wide star formation rate may be particularly sensitive to whether the central gaseous structure (typically a clumpy disk) is violently self-gravitating (Dekel et al. 2009; Ceverino et al. 2010). Star formation is most efficient when large gas clumps become self-gravitating, and large self-gravitating clumps of gas have been observed to form stars with high efficiency at $z \sim 3$ (e.g., Genzel et al. 2011).

Another possibility is that we are witnessing the effect of the growth of the circumgalactic medium (CGM) around galaxies. Observations with the Cosmic Origins Spectrograph 
on HST have recently revealed circumgalactic ionized gas halos at low redshift containing significant baryon and synthesized metal fractions (Werk et al. 2014). The CGM likely grew over time, as shock waves driven by supernova blast waves and superbubbles raised an increasing cumulative gas fraction to high temperatures. This reprocessed gas would be deposited in a warm ionized CGM and, at least for a period of time, off limits to conversion into the cold phase. However, the physics of the CGM is poorly understood, and in particular it is not clear at what rate the CGM gas recondenses back into the warm neutral phase in the disk.

Finally, the feedback which builds the CGM may be less efficient at high redshift, further increasing the amount of cold gas available for star formation. One may expect that the effects of feedback are less significant at high redshift where halos are denser and at fixed mass have higher circular velocities. Both make it more difficult for SNe to power galactic outflows that eject material into the CGM or outside the halo. Using the virial estimate from Bryan \& Norman (1998) we calculated the circular velocities of the host halos in our galaxy sample to be 242, 219, 219 and $197 \mathrm{~km} \mathrm{~s}^{-1}$ at $z=$ 4, 5, 6 and 7, respectively. Thus, the circular velocities in fact decrease towards higher redshift, which would if anything make it easier to eject material at higher redshift. However, the properties of the feedback mechanisms may evolve as well. First, as galaxies evolve and the typical gas density decreases, the supernova remnant thermalization efficiency, which is an increasing function of the cooling time in $\mathrm{H}$ II regions shock-heated by supernova blastwaves, increases, i.e., a progressively smaller fraction of the initial $\sim 10^{51}$ erg per supernova is quickly irradiated (see, e.g., Creasey et al. 2013). This implies that at lower redshifts, a larger fraction of supernova energy may be available to drive galaxy-wide outflows, thus making the feedback from star formation more effective. It is also possible that due to the very short growth times at high redshifts, so much gas is piling on that the outflows are somehow bottled in. Furthermore, the higher dust content at lower redshift can lead to stronger momentum-driven radiative stellar feedback, regulating further star formation (see, e.g., Murray et al. 2010; Andrews \& Thompson 2011).

One additional potential physical mechanism which may evolve is the ability of feedback from active galactic nuclei (AGNs) to suppress star formation, which is commonly implemented in theoretical models to avoid an overabundance of bright/massive galaxies (see discussion in Somerville \& Davé 2014). This type of feedback requires an accreting supermassive black hole, and although there are some examples of bright AGNs at very high redshift, the AGN/quasar luminosity function appears to decrease rapidly at $z>3$ (e.g., Richards et al. 2006). Bowler et al. (2015) have recently observed that the bright end of the galaxy UV luminosity function was steeper than the halo mass function at $z=6$, but not at $z=7$, and hypothesized that such an observation could be explained if feedback in bright/massive galaxies due to AGN first "turned on" at $z \lesssim 6$. However, the details of how AGN couple with galaxies and their surroundings, particularly at these epochs, are highly uncertain, so it remains unclear whether black hole accretion has significantly affected the growth of the galaxies we consider here.

While the scenarios we have discussed are clearly speculative, our observations imply that the latter effects, primarily a reduced efficiency of feedback at higher redshift due to a variety of redshift-dependent effects, control the evolution of the stellar baryon fraction. We conclude that the true cause of how a larger fraction of the baryons turns into stars at higher redshifts is most certainly determined by a delicate competition of factors.

Regardless of the underlying cause, the consequences of the increased availability of cold gas are intriguing. Our results at $z=6$ and 7 show that $\sim 10 \%-12 \%$ of the cosmic complement of baryons in these galaxies has been converted into stars. The remaining baryons must exist in the gas phase. If they are in the warm or cold neutral phase or the molecular phase, then the gas fraction in these phases is much higher than at lower redshifts (Magdis et al. 2012). A high gas mass fraction at very high redshifts is not unexpected and may soon be confirmed the Atacama Large Millimeter Array (ALMA; e.g., by measuring the dynamical mass via spectrally resolved FIR emission lines). There are indirect empirical hints that neutral and molecular gas fractions increase with redshift. Papovich et al. (2011) studied the evolution of observed galaxy SFRs, stellar masses, and sizes, and concluded that the gas-to-stellar mass fraction must rise with redshift, reaching $M_{\text {gas }} / M_{*}=3.9$ at $z=7$ (at a higher cumulative number density of $2 \times 10^{-4} \mathrm{Mpc}^{-3}$ ). This could intriguingly play some role in the decreasing visibility of $\operatorname{Ly} \alpha$ at $z>6$ (e.g., Fontana et al. 2010; Pentericci et al. 2011; Tilvi et al. 2014; Pentericci et al. 2014).

Finally, we examine the likely descendants of these bright $z=4-7$ galaxies. Specifically, being among the most rapidly star forming galaxies at their redshifts, could some of them end up evolving into extreme systems such as sub-millimeter galaxies (SMGs) by lower redshifts? Using the halo mass evolution tool from Behroozi et al. (2013a), we calculated the $68 \%$ confidence range of the descendant halo masses of our sample of bright high-redshift galaxies. We find that by $z=2$, galaxies we observe at $z=4,5,6$, and 7 will exist in halos with $\log \left(M_{\mathrm{h}} / M_{\odot}\right)=12.3-12.8,12.3-12.8,12.4-13$, and 12.4 13.1, respectively. SMGs are thought to be hosted by halos with $\log \left(M_{\mathrm{h}} / M_{\odot}\right) \approx 13$ (e.g., Hickox et al. 2012; Casey et al. 2014), thus the majority of $M_{\mathrm{UV}}^{*}$ galaxies at $z=4-5$ will evolve into galaxies at $z=2$ with halos slightly less massive than those of the typical SMGs. However, the SMG host halo mass begins to be consistent with the expected descendants of the $z=6-7$ galaxies, thus some subset of very UV-bright galaxies at $z>6$ may, in principle, turn into lower-redshift SMGs. A basic test of this is to see whether, if we assume these galaxies keep their current SFRs, they can grow large enough to match the stellar mass of a typical SMG by $z=2$. If we assume a SFR of $50 M_{\odot} \mathrm{yr}^{-1}$ (Table 1), we find that the $z=2$ descendants of $M_{\mathrm{UV}}^{*}$ galaxies at $z=6$ and 7 will have $\log \left(M_{*} / M_{\odot}\right)=11.1$. This is in the range of SMG stellar masses found in the literature (see review in Casey et al. 2014). Our assumed constant SFR of $\sim 50 M_{\odot} \mathrm{yr}^{-1}$, which implies stellar masses consistent with those observed in SMGs, is approximately the SFR disk galaxies should have prior to coalescence to produce SMGs in the merger-driven scenario for SMG formation (Narayanan et al. 2010).

\section{CONCLUSIONS}

Recent observations have shown that the characteristic UV luminosity $M_{\mathrm{UV}}^{*}$ does not significantly evolve from $z=4$ to 7 , which is unexpected given the general decline in the cosmic SFR density towards higher redshift over that time. To investigate the physical effects behind this observed non-evolution in $M_{\mathrm{UV}}^{*}$, we have inspected the stellar populations in $M_{\mathrm{UV}}^{*}$ galax- 
ies at $z=4$ to 7 . We have found the following results:

- Galaxies with $M_{\mathrm{UV}}<-21$ appear to have very similar physical properties at $z=4,5,6$, and 7 , including stellar mass, dust attenuation and SFR.

- Using abundance matching to infer the likely hosting halo masses, we found that $M_{\mathrm{UV}}<-21$ galaxies, which we have measured to have $\log \left(M_{*} / M_{\odot}\right)=9.6-9.9$, live in progressively smaller halos towards higher redshift, with $\log \left(M_{\mathrm{h}} / M_{\odot}\right)=11.93$ at $z=4$, and $\log \left(M_{\mathrm{h}} / M_{\odot}\right)=$ 11.35 at $z=7$.

- The stellar baryon fraction, defined as the fraction of baryons in stars in units of the cosmic mean $\Omega_{\mathrm{b}} / \Omega_{\mathrm{m}}$ rises from $0.051 \pm 0.006$ at $z=4$ to $0.117 \pm 0.043$ at $z=7$. This trend is significant at the $3.2 \sigma$ level.

- The observed trend of an increasing SBF with increasing redshift does not agree with simple expectations of how galaxies grow. Therefore, our observations imply a change in the physical properties governing starformation at $z>4$, such as, for example, a reduced efficiency of stellar and supernova feedback toward higher redshifts.

Future studies can improve upon our results by probing a larger volume to increase the sample of bright galaxies, allowing us to establish the evolution of the SBF at greater significance, as well as extending this analysis to $z=8$ and 9. Additionally, a more robust determination of the ratio between stellar mass and halo mass, and thus the SBF, can be done with accurate stellar mass functions, which are only now being computed at $z \geq 6$. Finally, through ALMA followup of distant galaxies, we will begin to not only directly probe their dust emission, removing some of the potential systematic biases inherent when assuming a dust attenuation curve, but ALMA can also directly probe the evolution of galaxy gas reservoirs with redshift. A direct observation of increasing cold gas reservoirs with increasing redshift would provide a complementary observation pointing to decreased feedback at high redshift leading to an increased stellar baryon fraction.

We thank Caitlin Casey for useful discussions on the abundance of dusty galaxies at high redshift. SLF acknowledges support from the University of Texas at Austin College of Natural Sciences. SLF and MS were also supported by a NASA Astrophysics and Data Analysis Program award issued by JPL/Caltech. MM acknowledges support from NSF grant AST-1413501. Partial support for DN was provided by NSF grants AST-1009452, AST-1442650, NASA grant HST-AR13906.001. This work is based on observations made with the NASA/ESA Hubble Space Telescope, obtained at the Space Telescope Science Institute, which is operated by the Association of Universities for Research in Astronomy, Inc., under NASA contract NAS 5-26555. These observations are associated with program \#12060. This work is also based in part on observations made with the Spitzer Space Telescope, which is operated by the Jet Propulsion Laboratory, California Institute of Technology under a contract with NASA. Support for this work was provided by NASA through an award issued by JPL/Caltech.

\section{REFERENCES}

Andrews, B. H., \& Thompson, T. A. 2011, ApJ, 727, 97

Ashby, M. L. N., Willner, S. P., Fazio, G. G., et al. 2013, ApJ, 769, 80 - 2015, ApJ Submitted

Barone-Nugent, R. L., Wyithe, J. S. B., Trenti, M., et al. 2015, ArXiv e-prints

Barone-Nugent, R. L., Trenti, M., Wyithe, J. S. B., et al. 2014, ApJ, 793, 17

Behroozi, P. S., Conroy, C., \& Wechsler, R. H. 2010, ApJ, 717, 379

Behroozi, P. S., Marchesini, D., Wechsler, R. H., et al. 2013a, ApJ, 777, L10

Behroozi, P. S., Wechsler, R. H., \& Conroy, C. 2013b, ApJ, 770, 57

Bouché, N., Dekel, A., Genzel, R., et al. 2010, ApJ, 718, 1001

Bouwens, R. J., Illingworth, G. D., Franx, M., \& Ford, H. 2007, ApJ, 670, 928

Bouwens, R. J., Illingworth, G. D., Oesch, P. A., et al. 2010, ApJ, 709, L133

-. 2011a, ArXiv e-prints

-. 2011b, ApJ, 737, 90

-. 2012, ApJ, 754, 83

-. 2014, ApJ, 793, 115

-. 2015, ApJ, 803, 34

Bowler, R. A. A., Dunlop, J. S., McLure, R. J., et al. 2014, MNRAS, 440, 2810

-. 2015, ArXiv e-prints

Bruzual, G., \& Charlot, S. 2003, MNRAS, 344, 1000

Bryan, G. L., \& Norman, M. L. 1998, ApJ, 495, 80

Calzetti, D., Armus, L., Bohlin, R. C., et al. 2000, ApJ, 533, 682

Capak, P. L., Carilli, C., Jones, G., et al. 2015, Nature, 522, 455

Casey, C. M., Narayanan, D., \& Cooray, A. 2014, Phys. Rep., 541, 45

Casey, C. M., Chen, C.-C., Cowie, L. L., et al. 2013, MNRAS, 436, 1919

Ceverino, D., Dekel, A., \& Bournaud, F. 2010, MNRAS, 404, 2151

Chapman, S. C., Blain, A. W., Smail, I., \& Ivison, R. J. 2005, ApJ, 622, 772

Creasey, P., Theuns, T., \& Bower, R. G. 2013, MNRAS, 429, 1922

Dekel, A., Zolotov, A., Tweed, D., et al. 2013, ArXiv e-prints

Dekel, A., Birnboim, Y., Engel, G., et al. 2009, Nature, 457, 451

Fakhouri, O., Ma, C.-P., \& Boylan-Kolchin, M. 2010, MNRAS, 406, 2267

Fazio, G. G., Hora, J. L., Allen, L. E., et al. 2004, ApJS, 154, 10

Finkelstein, S. L., Papovich, C., Giavalisco, M., et al. 2010, ApJ, 719, 1250

Finkelstein, S. L., Papovich, C., Ryan, R. E., et al. 2012a, ApJ, 758, 93
Finkelstein, S. L., Papovich, C., Salmon, B., et al. 2012b, ApJ, 756, 164 Finkelstein, S. L., Papovich, C., Dickinson, M., et al. 2013, Nature, 502, 524 Finkelstein, S. L., Ryan, Jr., R. E., Papovich, C., et al. 2015, ApJ, 810, 71 Finlator, K., Oppenheimer, B. D., \& Davé, R. 2011, MNRAS, 410, 1703 Fontana, A., Vanzella, E., Pentericci, L., et al. 2010, ApJ, 725, L205 Genzel, R., Newman, S., Jones, T., et al. 2011, ApJ, 733, 101 Gerke, B. F., Wechsler, R. H., Behroozi, P. S., et al. 2013, ApJS, 208, 1 Hickox, R. C., Wardlow, J. L., Smail, I., et al. 2012, MNRAS, 421, 284 Inoue, A. K. 2011, MNRAS, 415, 2920

Jaacks, J., Finkelstein, S. L., \& Nagamine, K. 2015, ArXiv e-prints

Jaacks, J., Nagamine, K., \& Choi, J. H. 2012, MNRAS, 427, 403

Kennicutt, Jr., R. C. 1998, ARA\&A, 36, 189

Klypin, A. A., Trujillo-Gomez, S., \& Primack, J. 2011, ApJ, 740, 102

Koekemoer, A. M., Faber, S. M., Ferguson, H. C., et al. 2011, ApJS, 197, 36 Komatsu, E., Smith, K. M., Dunkley, J., et al. 2011, ApJS, 192, 18

Kriek, M., Labbé, I., Conroy, C., et al. 2010, ApJ, 722, L64

Krumholz, M. R., \& Dekel, A. 2012, ApJ, 753, 16

Krumholz, M. R., Dekel, A., \& McKee, C. F. 2012, ApJ, 745, 69

Labbé, I., Oesch, P. A., Bouwens, R. J., et al. 2013, ApJ, 777, L19

Lee, K.-S., Giavalisco, M., Conroy, C., et al. 2009, ApJ, 695, 368

Lee, K.-S., Giavalisco, M., Gnedin, O. Y., et al. 2006, ApJ, 642, 63

Lee, S.-K., Ferguson, H. C., Somerville, R. S., Wiklind, T., \& Giavalisco, M. 2010, ApJ, 725, 1644

Leja, J., van Dokkum, P., \& Franx, M. 2013, ApJ, 766, 33

Madau, P. 1995, ApJ, 441, 18

Madau, P., \& Dickinson, M. 2014, ArXiv e-prints

Madau, P., Ferguson, H. C., Dickinson, M. E., et al. 1996, MNRAS, 283, 1388

Magdis, G. E., Daddi, E., Sargent, M., et al. 2012, ApJ, 758, L9

Mason, C. A., Treu, T., Schmidt, K. B., et al. 2015, ArXiv e-prints

McLure, R. J., Dunlop, J. S., Cirasuolo, M., et al. 2010, MNRAS, 403, 960

McLure, R. J., Dunlop, J. S., Bowler, R. A. A., et al. 2013, MNRAS, 432, 2696

Merlin, E., Fontana, A., Ferguson, H. C., et al. 2015, ArXiv e-prints

Moster, B. P., Somerville, R. S., Maulbetsch, C., et al. 2010, ApJ, 710, 903

Murray, N., Quataert, E., \& Thompson, T. A. 2010, ApJ, 709, 191 
Narayanan, D., Hayward, C. C., Cox, T. J., et al. 2010, MNRAS, 401, 1613 Neistein, E., van den Bosch, F. C., \& Dekel, A. 2006, MNRAS, 372, 933 Oesch, P. A., Bouwens, R. J., Illingworth, G. D., et al. 2010, ApJ, 709, L16 —. 2012, ApJ, 759, 135

-. 2013, ApJ, 773, 75

-. 2014, ApJ, 786, 108

Oke, J. B., \& Gunn, J. E. 1983, ApJ, 266, 713

Ono, Y., Ouchi, M., Mobasher, B., et al. 2012, ApJ, 744, 83

Overzier, R. A., Bouwens, R. J., Illingworth, G. D., \& Franx, M. 2006, ApJ, 648, L5

Papovich, C., Dickinson, M., \& Ferguson, H. C. 2001, ApJ, 559, 620

Papovich, C., Finkelstein, S. L., Ferguson, H. C., Lotz, J. M., \& Giavalisco, M. 2011, MNRAS, 412, 1123

Pei, Y. C. 1992, ApJ, 395, 130

Pentericci, L., Fontana, A., Vanzella, E., et al. 2011, ApJ, 743, 132

Pentericci, L., Vanzella, E., Fontana, A., et al. 2014, ArXiv e-prints

Reddick, R. M., Wechsler, R. H., Tinker, J. L., \& Behroozi, P. S. 2013, ApJ, 771,30

Reddy, N., Dickinson, M., Elbaz, D., et al. 2012a, ApJ, 744, 154

Reddy, N. A., Pettini, M., Steidel, C. C., et al. 2012b, ApJ, 754, 25

Reddy, N. A., \& Steidel, C. C. 2009, ApJ, 692, 778

Reddy, N. A., Steidel, C. C., Erb, D. K., Shapley, A. E., \& Pettini, M. 2006, ApJ, 653, 1004
Richards, G. T., Strauss, M. A., Fan, X., et al. 2006, AJ, 131, 2766 Salmon, B., Papovich, C., Finkelstein, S. L., et al. 2015, ApJ, 799, 183 Schechter, P. 1976, ApJ, 203, 297

Schenker, M. A., Robertson, B. E., Ellis, R. S., et al. 2013, ApJ, 768, 196 Somerville, R. S., \& Davé, R. 2014, ArXiv e-prints

Somerville, R. S., Hopkins, P. F., Cox, T. J., Robertson, B. E., \& Hernquist, L. 2008, MNRAS, 391, 481

Song, M., Finkelstein, S. L., Ashby, M. L. N., et al. 2015, ArXiv e-prints Tilvi, V., Papovich, C., Tran, K.-V. H., et al. 2013, ApJ, 768, 56

Tilvi, V., Papovich, C., Finkelstein, S. L., et al. 2014, ArXiv e-prints

Tinker, J., Kravtsov, A. V., Klypin, A., et al. 2008, ApJ, 688, 709

van Dokkum, P. G., Whitaker, K. E., Brammer, G., et al. 2010, ApJ, 709, 1018

Watson, D., Christensen, L., Knudsen, K. K., et al. 2015, Nature, 519, 327

Werk, J. K., Prochaska, J. X., Tumlinson, J., et al. 2014, ApJ, 792, 8

Wilkins, S. M., Bunker, A. J., Lorenzoni, S., \& Caruana, J. 2011, MNRAS, 411, 23

Wolfire, M. G., Hollenbach, D., McKee, C. F., Tielens, A. G. G. M., \& Bakes, E. L. O. 1995, ApJ, 443, 152

Wolfire, M. G., McKee, C. F., Hollenbach, D., \& Tielens, A. G. G. M. 2003, ApJ, 587, 278

APPENDIX

In the above text, we discuss the median stellar population properties of our sample of galaxies. Galaxies of course are an inhomogeneous population, thus in this appendix we provide tables of the individual properties for each of the bright galaxies used in our analysis (those with IRAC measurements, and a best-fitting SED model with $\chi^{2}<20$; see $\S 2.3$ ). Each column in these tables lists the best-fitting value along with the $68 \%$ confidence range in parentheses.

TABLE A1

Stellar Populations of Bright Galaxies at $z=4$

\begin{tabular}{|c|c|c|c|c|c|c|c|}
\hline ID & $\begin{array}{c}\text { Right Ascension } \\
\text { (J2000) }\end{array}$ & $\begin{array}{c}\text { Declination } \\
(\mathrm{J} 2000)\end{array}$ & Redshift & $\begin{array}{c}\log \mathrm{M}_{*} \\
\left(\mathrm{M}_{\odot}\right)\end{array}$ & $\begin{array}{l}\text { Age } \\
(\mathrm{Myr})\end{array}$ & $\mathrm{E}(\mathrm{B}-\mathrm{V})$ & $\begin{array}{c}\text { SFR } \\
\left(\mathrm{M}_{\odot} \mathrm{yr}^{-1}\right)\end{array}$ \\
\hline z4_GSD_34736 & 53.096840 & -27.866074 & $3.51(3.44-3.66)$ & $9.97(10.13-10.49)$ & $10(19-101)$ & $0.34(0.18-0.34)$ & $451(109-434)$ \\
\hline z4_GSD_30292 & 53.086891 & -27.844139 & $3.51(3.46-3.75)$ & $10.49(10.31-10.51)$ & $57(49-90)$ & $0.24(0.16-0.32)$ & $132(64-322)$ \\
\hline z4_GNW_9013 & 189.085114 & 62.160465 & $3.54(3.49-3.60)$ & $9.97(9.93-10.15)$ & $1015(202-1015)$ & $0.10(0.02-0.10)$ & $54(26-53)$ \\
\hline z4_ERS_4095 & 53.143330 & -27.690090 & $3.55(3.48-3.67)$ & $10.27(10.17-10.55)$ & $49(30-101)$ & $0.16(0.14-0.28)$ & $88(73-299)$ \\
\hline z4_ERS_9969 & 53.120926 & -27.709446 & $3.57(3.52-3.64)$ & $9.68(9.43-9.68)$ & $57(30-286)$ & $0.04(0.00-0.10)$ & $20(14-38)$ \\
\hline z4_GNW_26176 & 189.483200 & 62.284786 & $3.63(3.57-3.71)$ & $9.99(9.96-10.18)$ & $90(40-202)$ & $0.12(0.02-0.14)$ & $65(27-85)$ \\
\hline z4_GNW_1986 & 189.159058 & 62.115471 & $3.64(3.58-3.70)$ & $9.98(9.96-10.19)$ & $101(49-1015)$ & $0.08(0.06-0.10)$ & $55(44-77)$ \\
\hline z4_ERS_16929 & 53.087231 & -27.729538 & $3.64(3.58-3.72)$ & $9.42(9.21-9.85)$ & $19(10-40)$ & $0.14(0.14-0.20)$ & $59(55-109)$ \\
\hline z4_GSD_29028 & 53.087368 & -27.839535 & $3.64(3.52-3.75)$ & $10.17(10.12-10.22)$ & $80(80-101)$ & $0.10(0.06-0.10)$ & $38(25-40)$ \\
\hline z4_ERS_20075 & 53.020580 & -27.742151 & $3.65(3.59-3.71)$ & $10.55(10.54-10.66)$ & $202(202-570)$ & $0.06(0.02-0.08)$ & $75(53-104)$ \\
\hline z4_GND_30689 & 189.339355 & 62.216450 & $3.66(3.59-3.75)$ & $9.45(9.40-9.70)$ & $39(30-71)$ & $0.04(0.00-0.10)$ & $20(14-36)$ \\
\hline z4_GSW_4356 & 53.109478 & -27.879360 & $3.67(3.61-3.77)$ & $9.72(9.66-9.90)$ & $57(49-90)$ & $0.00(0.00-0.08)$ & $19(19-43)$ \\
\hline z4_GSD_15786 & 53.071735 & -27.798437 & $3.67(3.60-3.75)$ & $9.61(9.67-9.88)$ & $49(57-1015)$ & $0.04(0.00-0.10)$ & $19(14-33)$ \\
\hline z'4_GSD_535 & 53.198959 & -27.737940 & $3.69(3.57-3.81)$ & $9.59(9.53-9.79)$ & $49(40-57)$ & $0.00(0.00-0.08)$ & $18(17-39)$ \\
\hline z4_ERS_3396 & 53.117710 & -27.686771 & $3.69(3.64-3.75)$ & $9.90(9.53-9.90)$ & $49(30-49)$ & $0.10(0.08-0.16)$ & $48(40-74)$ \\
\hline z4_GSD_21002 & 53.121414 & -27.814621 & $3.69(3.63-3.76)$ & $9.80(9.57-9.85)$ & $30(10-30)$ & $0.18(0.16-0.24)$ & $93(70-152)$ \\
\hline z4_GSD_35257 & 53.107422 & -27.869299 & $3.70(3.58-3.82)$ & $10.46(10.24-10.47)$ & $101(71-101)$ & $0.28(0.10-0.28)$ & $222(45-240)$ \\
\hline z4_ERS_11888 & 53.069328 & -27.714815 & $3.70(3.62-3.78)$ & $9.55(9.29-9.76)$ & $49(19-49)$ & $0.00(0.00-0.18)$ & $14(14-77)$ \\
\hline z4_GSD_11269 & 53.031239 & -27.785215 & $3.71(3.61-3.79)$ & $10.06(10.02-10.16)$ & $49(40-101)$ & $0.10(0.10-0.24)$ & $45(43-176)$ \\
\hline z4_GSD_27735 & 53.138859 & -27.835371 & $3.72(3.65-3.79)$ & $9.56(9.36-9.63)$ & $30(19-57)$ & $0.14(0.02-0.18)$ & $54(17-78)$ \\
\hline z4_GSW_2898 & 53.144775 & -27.871527 & $3.78(3.68-3.87)$ & $10.18(9.88-10.20)$ & $39(30-49)$ & $0.20(0.06-0.26)$ & $131(35-200)$ \\
\hline z4_GSD_31543 & 53.066261 & -27.849056 & $3.81(3.75-3.98)$ & $10.12(10.00-10.20)$ & $49(30-57)$ & $0.12(0.10-0.20)$ & $52(41-117)$ \\
\hline z4_GND_38889 & 189.181412 & 62.189281 & $3.81(3.75-4.05)$ & $10.40(10.38-10.48)$ & $57(49-71)$ & $0.14(0.14-0.22)$ & $68(65-163)$ \\
\hline z4_ERS_4079 & 53.110340 & -27.689985 & $3.81(3.74-3.99)$ & $10.09(9.87-10.17)$ & $19(10-57)$ & $0.32(0.06-0.34)$ & $325(31-373)$ \\
\hline z4_GSW_7015 & 53.189873 & -27.892590 & $3.83(3.75-3.93)$ & $10.15(9.77-10.15)$ & $49(30-80)$ & $0.16(0.06-0.22)$ & $84(32-152)$ \\
\hline z4_GSW_6936 & 53.073589 & -27.892235 & $3.83(3.76-3.92)$ & $10.47(10.40-10.49)$ & $286(202-718)$ & $0.00(0.00-0.12)$ & $22(22-81)$ \\
\hline z4_GSW_9851 & 53.181850 & -27.906641 & $3.85(3.79-3.95)$ & $9.97(9.64-9.94)$ & $49(30-49)$ & $0.12(0.00-0.20)$ & $56(18-114)$ \\
\hline z4_GNW_12987 & 189.040390 & 62.186352 & $3.92(3.84-4.00)$ & $9.66(9.49-9.69)$ & $57(40-71)$ & $0.02(0.00-0.02)$ & $19(15-23)$ \\
\hline z4_ERS_5026 & 53.133690 & -27.693453 & $3.93(3.82-4.05)$ & $9.67(9.63-9.90)$ & $39(30-101)$ & $0.06(0.00-0.12)$ & $27(16-49)$ \\
\hline z4_GSD_34857 & 53.076183 & -27.866360 & $3.98(3.89-4.11)$ & $10.32(10.23-10.37)$ & $286(40-286)$ & $0.16(0.14-0.16)$ & $123(96-128)$ \\
\hline z4_GSD_23593 & 53.232452 & -27.822868 & $3.99(3.89-4.14)$ & $9.48(9.33-9.92)$ & $19(19-80)$ & $0.18(0.14-0.22)$ & $72(52-101)$ \\
\hline z'4_GSD_905 & 53.168266 & -27.741940 & $4.01(3.92-4.11)$ & $9.78(9.44-9.76)$ & $90(19-90)$ & $0.00(0.00-0.20)$ & $13(13-88)$ \\
\hline z4_GNNW_25081 & 189.331039 & 62.290836 & $4.01(3.90-4.10)$ & $9.39(9.34-9.43)$ & $10(10-10)$ & $0.26(0.24-0.26)$ & $140(122-144)$ \\
\hline z4_ERS_3543 & 53.144386 & -27.687588 & $4.02(3.87-4.16)$ & $10.01(9.96-10.26)$ & $39(40-404)$ & $0.16(0.06-0.16)$ & $78(30-83)$ \\
\hline z4_GND_40720 & 189.179291 & 62.182003 & $4.02(3.84-4.20)$ & $9.70(9.26-9.74)$ & $30(19-80)$ & $0.20(0.02-0.20)$ & $75(12-83)$ \\
\hline z4_GNW_18460 & 189.286835 & 62.367325 & $4.05(3.92-4.16)$ & $10.28(10.22-10.38)$ & $286(202-806)$ & $0.00(0.00-0.10)$ & $20(19-53)$ \\
\hline z4_ERS_22264 & 53.075272 & -27.755194 & $4.06(3.96-4.17)$ & $10.12(10.01-10.24)$ & $39(30-508)$ & $0.16(0.10-0.16)$ & $93(62-99)$ \\
\hline z4_GND_27047 & 189.356354 & 62.227554 & $4.10(3.92-4.21)$ & $10.47(10.26-10.54)$ & $202(49-904)$ & $0.12(0.12-0.20)$ & $52(50-98)$ \\
\hline z4_GNW_21799 & 189.329178 & 62.331532 & $4.10(4.03-4.17)$ & $9.82(9.69-9.96)$ & $57(40-80)$ & $0.00(0.00-0.04)$ & $24(23-35)$ \\
\hline z4_GNW_23907 & 189.467804 & 62.297764 & $4.10(3.98-4.21)$ & $9.94(9.82-10.31)$ & $80(19-101)$ & $0.20(0.14-0.24)$ & $122(79-164)$ \\
\hline
\end{tabular}


Increasing Stellar Baryon Fractions at $z>4$

TABLE A1

Stellar Populations of BRIGHT Galaxies at $z=4$

\begin{tabular}{|c|c|c|c|c|c|c|c|}
\hline z4_GND_7728 & 189.270905 & 62.291943 & $4.10(4.01-4.20)$ & $10.23(9.78-10.24)$ & $101(19-101)$ & $0.20(0.16-0.22)$ & $133(91-163)$ \\
\hline z4_GSD_15152 & 53.027557 & -27.796583 & $4.12(4.05-4.22)$ & $10.41(10.23-10.44)$ & $101(49-101)$ & $0.20(0.20-0.24)$ & $171(178-266)$ \\
\hline z4_GNW_17778 & 189.311584 & 62.382095 & $4.14(4.00-4.29)$ & $9.76(9.29-9.82)$ & $30(19-49)$ & $0.18(0.10-0.20)$ & $85(41-99)$ \\
\hline z4_ERS_11615 & 53.107601 & -27.713976 & $4.14(4.01-4.26)$ & $9.52(9.61-9.96)$ & $19(30-101)$ & $0.18(0.02-0.18)$ & $79(18-83)$ \\
\hline z4_GND_23790 & 189.233093 & 62.236786 & $4.15(4.06-4.27)$ & $10.50(10.49-10.60)$ & $101(57-404)$ & $0.18(0.08-0.18)$ & $211(83-221)$ \\
\hline z4_GSD_20508 & 53.192692 & -27.813051 & $4.17(4.06-4.26)$ & $9.65(9.61-9.92)$ & $30(30-202)$ & $0.14(0.04-0.16)$ & $59(25-76)$ \\
\hline z4_GNW_18340 & 189.299255 & 62.370079 & $4.19(4.03-4.30)$ & $11.29(11.17-11.33)$ & $1015(904-1015)$ & $0.18(0.18-0.24)$ & $146(142-226)$ \\
\hline z4_GNW_15232 & 189.014038 & 62.200378 & $4.22(4.11-4.31)$ & $10.18(9.80-10.18)$ & $71(30-80)$ & $0.22(0.20-0.26)$ & $178(139-242)$ \\
\hline z4_ERS_14762 & 53.016903 & -27.723013 & $4.22(4.11-4.32)$ & $9.40(9.32-9.84)$ & $19(19-80)$ & $0.16(0.08-0.18)$ & $57(28-78)$ \\
\hline z4_GNW_11056 & 189.013092 & 62.173153 & $4.23(4.08-4.31)$ & $9.64(9.61-9.78)$ & $39(30-57)$ & $0.04(0.00-0.08)$ & $26(16-38)$ \\
\hline z4_GSW_66 & 53.230198 & -27.839573 & $4.24(4.17-4.33)$ & $9.76(9.76-10.22)$ & $19(19-90)$ & $0.18(0.12-0.18)$ & $138(80-143)$ \\
\hline z4_GSD_9138 & 53.215435 & -27.778782 & $4.24(4.10-4.37)$ & $10.07(9.82-10.08)$ & $101(80-101)$ & $0.18(0.00-0.20)$ & $92(18-102)$ \\
\hline z4_GNW_3896 & 189.145218 & 62.129818 & $4.24(4.12-4.39)$ & $9.80(9.77-10.12)$ & $10(10-40)$ & $0.34(0.32-0.34)$ & $346(297-359)$ \\
\hline z4_GNW_8115 & 189.145187 & 62.154770 & $4.25(4.09-4.34)$ & $9.62(9.54-9.79)$ & $49(40-80)$ & $0.02(0.00-0.06)$ & $19(15-31)$ \\
\hline z4_GNW_26546 & 189.445068 & 62.281898 & $4.26(4.02-4.38)$ & $10.36(10.35-10.59)$ & $101(57-286)$ & $0.28(0.00-0.30)$ & $151(12-228)$ \\
\hline z4_GND_9704 & 189.201706 & 62.278599 & $4.27(4.19-4.36)$ & $9.85(9.85-10.23)$ & $30(30-90)$ & $0.14(0.04-0.16)$ & $95(38-117)$ \\
\hline z4_GND_21065 & 189.290955 & 62.244240 & $4.29(4.13-4.41)$ & $10.47(10.17-10.53)$ & $202(80-286)$ & $0.30(0.18-0.30)$ & $264(81-240)$ \\
\hline z4_GND_31952 & 189.119812 & 62.212612 & $4.29(4.19-4.37)$ & $9.56(9.50-9.90)$ & $30(30-57)$ & $0.12(0.06-0.14)$ & $45(27-58)$ \\
\hline z4_GNW_24624 & 189.388474 & 62.294460 & $4.30(4.17-4.43)$ & $9.34(9.35-9.62)$ & $39(30-57)$ & $0.00(0.00-0.10)$ & $12(11-41)$ \\
\hline z4_GNW_11698 & 188.988419 & 62.177494 & $4.30(4.19-4.39)$ & $9.62(9.42-9.66)$ & $30(19-49)$ & $0.16(0.02-0.20)$ & $61(14-82)$ \\
\hline 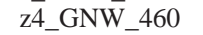 & 189.120911 & 62.101513 & $4.30(4.18-4.40)$ & $9.41(9.36-9.80)$ & $30(19-71)$ & $0.08(0.00-0.16)$ & $27(14-64)$ \\
\hline z4_GNW_2957 & 189.185181 & 62.122967 & $4.30(4.17-4.42)$ & $9.90(9.89-10.12)$ & $30(30-49)$ & $0.16(0.12-0.18)$ & $84(59-95)$ \\
\hline z4_GNW_31531 & 189.295654 & 62.349960 & $4.31(4.15-4.43)$ & $10.32(10.10-10.46)$ & $404(49-286)$ & $0.24(0.16-0.30)$ & $122(54-208)$ \\
\hline z4_GNW_7377 & 189.108002 & 62.151005 & $4.31(4.25-4.37)$ & $10.35(10.24-10.35)$ & $80(30-80)$ & $0.00(0.00-0.12)$ & $57(56-179)$ \\
\hline z4_GSD_2 1252 & 53.143112 & -27.815502 & $4.33(4.24-4.39)$ & $10.05(10.03-10.47)$ & $57(30-101)$ & $0.18(0.16-0.22)$ & $181(149-266)$ \\
\hline z4_GND_30347 & 189.324326 & 62.217796 & $4.34(4.24-4.41)$ & $9.71(9.54-9.82)$ & $1015(40-404)$ & $0.08(0.06-0.12)$ & $26(21-39)$ \\
\hline z4_GNW_24183 & 189.466507 & 62.296875 & $4.35(4.21-4.43)$ & $10.09(9.95-10.22)$ & $570(71-570)$ & $0.14(0.10-0.18)$ & $68(42-107)$ \\
\hline z4_GNW_21312 & 189.366592 & 62.330898 & $4.38(4.25-4.46)$ & $9.66(9.72-10.34)$ & $10(10-30)$ & $0.26(0.22-0.32)$ & $210(154-394)$ \\
\hline z4_GND_27301 & 189.093216 & 62.226814 & $4.39(4.29-4.47)$ & $10.00(9.90-10.14)$ & $904(40-718)$ & $0.14(0.10-0.18)$ & $50(37-74)$ \\
\hline z4_GSD_16522 & 53.076065 & -27.800694 & $4.39(4.32-4.47)$ & $9.67(9.52-10.14)$ & $19(10-90)$ & $0.22(0.22-0.26)$ & $160(154-224)$ \\
\hline z4_GNW_21830 & 189.366791 & 62.331589 & $4.40(4.30-4.48)$ & $9.95(9.95-10.26)$ & $30(30-286)$ & $0.20(0.14-0.20)$ & $118(65-120)$ \\
\hline z4_GND_7158 & 189.252747 & 62.298897 & $4.41(4.31-4.50)$ & $10.27(9.94-10.30)$ & $101(40-101)$ & $0.26(0.22-0.28)$ & $146(90-167)$ \\
\hline z4_GNW_32075 & 189.304321 & 62.353088 & $4.41(4.31-4.48)$ & $10.46(10.45-10.77)$ & $30(30-202)$ & $0.26(0.20-0.26)$ & $384(229-397)$ \\
\hline z"̄_GNW_7816 & 189.049530 & 62.152920 & $4.42(4.33-4.49)$ & $9.70(9.71-10.15)$ & $19(19-71)$ & $0.18(0.12-0.20)$ & $100(64-121)$ \\
\hline z4_GSW_5453 & 53.199787 & -27.884937 & $4.42(4.32-4.49)$ & $9.90(9.77-10.03)$ & $286(80-570)$ & $0.16(0.14-0.20)$ & $52(42-75)$ \\
\hline z4_GND_39360 & 189.242157 & 62.187439 & $4.44(4.32-4.54)$ & $9.86(9.79-10.15)$ & $71(30-90)$ & $0.18(0.16-0.24)$ & $65(54-118)$ \\
\hline z4_GSW_1319 & 53.201015 & -27.860250 & $4.44(4.37-4.51)$ & $9.84(9.60-9.87)$ & $39(30-90)$ & $0.14(0.00-0.18)$ & $60(16-89)$ \\
\hline z4_GNW_8416 & 189.195557 & 62.156864 & $4.45(4.31-4.54)$ & $10.51(10.39-10.58)$ & $286(49-286)$ & $0.24(0.20-0.30)$ & $136(91-259)$ \\
\hline z4_GND_32575 & 189.173126 & 62.210808 & $4.45(4.36-4.53)$ & $9.75(9.37-9.72)$ & $39(30-49)$ & $0.14(0.06-0.18)$ & $49(21-72)$ \\
\hline z4_GNW_13552 & 189.061249 & 62.189472 & $4.45(4.34-4.54)$ & $9.92(9.94-10.38)$ & $19(19-71)$ & $0.24(0.20-0.26)$ & $164(109-184)$ \\
\hline z4_GNW_8070 & 189.107422 & 62.154560 & $4.45(4.37-4.53)$ & $9.81(9.71-9.88)$ & $30(30-49)$ & $0.16(0.04-0.18)$ & $96(29-105)$ \\
\hline z4_ERS_5818 & 53.069077 & -27.696518 & $4.47(4.38-4.56)$ & $9.43(9.37-9.64)$ & $39(30-49)$ & $0.02(0.00-0.16)$ & $16(13-65)$ \\
\hline z4_GNW_20572 & 189.313507 & 62.320702 & $4.47(4.37-4.56)$ & $9.83(9.52-9.86)$ & $39(30-90)$ & $0.16(0.04-0.16)$ & $58(17-56)$ \\
\hline z4_GNW_18301 & 189.335205 & 62.370796 & $4.47(4.39-4.54)$ & $9.97(9.88-10.31)$ & $30(30-90)$ & $0.18(0.16-0.22)$ & $116(98-194)$ \\
\hline z4_GNW_18575 & 189.297821 & 62.365429 & $4.49(4.42-4.55)$ & $10.70(10.37-10.74)$ & $30(19-57)$ & $0.28(0.24-0.30)$ & $739(458-894)$ \\
\hline z"̄_GNW_2261 & 189.192337 & 62.117081 & $4.50(4.42-4.57)$ & $9.96(9.62-9.97)$ & $39(30-80)$ & $0.14(0.02-0.18)$ & $79(23-115)$ \\
\hline z4_GND_40010 & 189.335739 & 62.184937 & $4.51(4.44-4.56)$ & $10.16(9.98-10.20)$ & $57(30-90)$ & $0.06(0.00-0.16)$ & $62(35-158)$ \\
\hline z4_GNW_7213 & 189.108322 & 62.150093 & $4.51(0.69-4.57)$ & $9.89(6.51-9.87)$ & $30(19-90)$ & $0.20(0.10-0.66)$ & $116(0-142)$ \\
\hline z4_GNW_7206 & 189.108185 & 62.149807 & $4.54(4.46-4.61)$ & $9.97(9.85-10.12)$ & $19(19-30)$ & $0.26(0.22-0.28)$ & $245(149-279)$ \\
\hline z4_GSW_8512 & 53.177471 & -27.900093 & $4.54(4.48-4.60)$ & $9.53(9.54-10.02)$ & $19(19-40)$ & $0.18(0.18-0.26)$ & $67(67-152)$ \\
\hline z4_GND_25942 & 189.147903 & 62.230583 & $4.54(4.44-4.63)$ & $10.09(9.89-10.23)$ & $19(19-57)$ & $0.30(0.24-0.32)$ & $325(180-360)$ \\
\hline z4_GSD_36028 & 53.079254 & -27.877260 & $4.54(4.46-4.59)$ & $10.21(9.96-10.22)$ & $1015(30-202)$ & $0.10(0.06-0.12)$ & $83(56-104)$ \\
\hline z4_GND_14271 & 189.305084 & 62.263287 & $4.56(4.44-4.65)$ & $10.79(10.45-10.79)$ & $39(40-80)$ & $0.36(0.30-0.36)$ & $537(295-541)$ \\
\hline Z4_GNW_18613 & 189.276077 & 62.364826 & $4.57(4.47-4.64)$ & $10.04(10.06-10.58)$ & $19(19-57)$ & $0.22(0.20-0.26)$ & $219(184-335)$ \\
\hline
\end{tabular}


TABLE A2

Stellar Populations of BRIGHT Galaxies at $z=5$

\begin{tabular}{|c|c|c|c|c|c|c|c|}
\hline ID & $\begin{array}{c}\text { Right Ascension } \\
\text { (J2000) }\end{array}$ & $\begin{array}{c}\text { Declination } \\
(\mathrm{J} 2000)\end{array}$ & Redshift & $\begin{array}{c}\log \mathrm{M}_{*} \\
\left(\mathrm{M}_{\odot}\right)\end{array}$ & $\begin{array}{l}\text { Age } \\
(\mathrm{Myr})\end{array}$ & $\mathrm{E}(\mathrm{B}-\mathrm{V})$ & $\begin{array}{c}\text { SFR } \\
\left(\mathrm{M}_{\odot} \mathrm{yr}^{-1}\right)\end{array}$ \\
\hline z5_GSW_8762 & 53.208008 & -27.901289 & $4.51(4.44-4.57)$ & $9.72(9.50-9.78)$ & $49(30-71)$ & $0.06(0.00-0.08)$ & $31(16-38)$ \\
\hline z5_GND_36639 & 189.186142 & 62.197327 & $4.56(4.45-4.68)$ & $10.25(10.11-10.52)$ & $80(19-101)$ & $0.28(0.26-0.32)$ & $223(203-330)$ \\
\hline z5_GNW_17976 & 189.300125 & 62.377483 & $4.57(4.42-4.74)$ & $10.19(10.12-10.37)$ & $286(80-286)$ & $0.22(0.10-0.26)$ & $102(31-164)$ \\
\hline z5_GSD_9044 & 53.091724 & -27.778580 & $4.58(4.48-4.67)$ & $10.26(10.02-10.25)$ & $202(40-508)$ & $0.06(0.06-0.18)$ & $32(33-110)$ \\
\hline z5_GSW_6918 & 53.234589 & -27.892109 & $4.66(4.58-4.75)$ & $9.52(9.38-9.59)$ & $57(49-57)$ & $0.00(0.00-0.02)$ & $14(13-18)$ \\
\hline z5_GND_38041 & 189.299210 & 62.192570 & $4.67(4.60-4.74)$ & $9.36(9.31-9.89)$ & $10(10-19)$ & $0.22(0.20-0.28)$ & $106(94-198)$ \\
\hline z5_GND_16758 & 189.176086 & 62.256329 & $4.71(4.62-4.84)$ & $10.03(9.96-10.11)$ & $19(19-30)$ & $0.26(0.26-0.28)$ & $277(272-336)$ \\
\hline z5_GSD_33149 & 53.070778 & -27.856453 & $4.71(4.62-4.81)$ & $10.02(9.63-10.30)$ & $101(10-508)$ & $0.20(0.10-0.24)$ & $116(49-172)$ \\
\hline z5_GSD_13326 & 53.095345 & -27.790989 & $4.71(4.64-4.79)$ & $10.19(9.98-10.26)$ & $30(19-80)$ & $0.26(0.16-0.26)$ & $228(86-238)$ \\
\hline z5_ERS_3475 & 53.070839 & -27.687143 & $4.72(4.66-4.81)$ & $9.95(9.84-10.39)$ & $19(19-40)$ & $0.26(0.26-0.32)$ & $205(211-369)$ \\
\hline z5_GND_38212 & 189.273590 & 62.192028 & $4.72(4.65-5.06)$ & $9.84(9.82-10.19)$ & $30(30-71)$ & $0.16(0.08-0.20)$ & $72(43-136)$ \\
\hline z5̄G_GSW_1565 & 53.238213 & -27.862486 & $4.75(4.70-4.79)$ & $9.96(9.51-9.96)$ & $30(19-30)$ & $0.18(0.12-0.20)$ & $135(73-158)$ \\
\hline z5_GND_12253 & 189.294418 & 62.269447 & $4.77(4.70-4.90)$ & $10.06(9.87-10.19)$ & $570(40-718)$ & $0.14(0.10-0.20)$ & $55(39-104)$ \\
\hline z5_ERS_12604 & 53.021912 & -27.716784 & $4.81(4.76-4.89)$ & $9.60(9.60-9.84)$ & $39(40-1015)$ & $0.08(0.02-0.10)$ & $30(17-34)$ \\
\hline z5_GNW_25539 & 189.489624 & 62.288536 & $4.81(4.76-4.87)$ & $10.34(10.03-10.43)$ & $19(19-40)$ & $0.26(0.18-0.26)$ & $570(266-567)$ \\
\hline z5_ERS_2517 & 53.119019 & -27.682158 & $4.82(4.76-4.93)$ & $9.70(9.41-9.77)$ & $30(30-80)$ & $0.18(0.00-0.16)$ & $73(14-68)$ \\
\hline z5_GND_17343 & 189.091400 & 62.254662 & $4.83(4.75-4.95)$ & $10.16(10.05-10.19)$ & $30(19-71)$ & $0.24(0.06-0.24)$ & $214(45-230)$ \\
\hline z'5_ERS_6044 & 53.048820 & -27.697111 & $4.85(4.78-4.94)$ & $9.85(9.78-9.87)$ & $90(30-90)$ & $0.00(0.00-0.20)$ & $15(15-94)$ \\
\hline z5_GSW_6966 & 53.245884 & -27.892273 & $4.94(0.83-4.98)$ & $10.29(10.20-10.37)$ & $19(10-30)$ & $0.34(0.28-0.36)$ & $506(265-566)$ \\
\hline z5_GNW_6112 & 189.064835 & 62.143963 & $4.95(4.90-5.06)$ & $9.85(9.47-10.02)$ & $19(10-71)$ & $0.26(0.06-0.28)$ & $183(31-221)$ \\
\hline z5_GSD_10352 & 53.021172 & -27.782366 & $4.95(4.91-4.99)$ & $10.43(10.36-10.80)$ & $57(19-90)$ & $0.22(0.08-0.24)$ & $474(137-571)$ \\
\hline z5_GNW_4779 & 189.203064 & 62.136204 & $4.96(4.88-5.15)$ & $9.86(10.07-10.31)$ & $19(30-286)$ & $0.28(0.04-0.32)$ & $174(20-244)$ \\
\hline z5_GND_15230 & 189.205124 & 62.260712 & $5.02(4.96-5.08)$ & $9.41(9.41-9.69)$ & $39(30-49)$ & $0.00(0.00-0.12)$ & $15(15-59)$ \\
\hline z5_GNW_16101 & 189.000168 & 62.207241 & $5.02(4.78-5.06)$ & $9.50(9.20-9.52)$ & $49(30-49)$ & $0.02(0.00-0.04)$ & $18(12-20)$ \\
\hline z5_GNW_10657 & 189.130219 & 62.170780 & $5.03(4.80-5.15)$ & 9.35 (9.19-9.44) & $49(30-57)$ & $0.00(0.00-0.06)$ & $13(11-27)$ \\
\hline z5_GNW_19973 & 189.371780 & 62.324554 & $5.03(4.80-5.12)$ & $10.10(10.15-10.59)$ & $10(10-202)$ & $0.38(0.22-0.34)$ & $681(140-473)$ \\
\hline 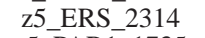 & 53.088764 & -27.680889 & $5.05(5.01-5.20)$ & $9.55(9.43-9.60)$ & $30(19-49)$ & $0.14(0.00-0.20)$ & $52(16-86)$ \\
\hline z5_PAR1_1735 & 53.246998 & -27.686445 & $5.07(4.99-5.18)$ & $10.06(9.74-10.09)$ & $49(30-71)$ & $0.16(0.08-0.18)$ & $69(32-79)$ \\
\hline z5_GNW_13254 & 189.039703 & 62.187725 & $5.24(5.15-5.32)$ & $9.88(9.74-9.93)$ & $71(49-71)$ & $0.00(0.00-0.04)$ & $22(21-33)$ \\
\hline z5_ERS_9511 & 53.155231 & -27.700718 & $5.26(5.05-5.40)$ & $9.90(9.49-9.99)$ & 49 (19-90) & $0.12(0.00-0.20)$ & $47(14-98)$ \\
\hline z5_GNW_25408 & 189.457428 & 62.289562 & $5.27(5.11-5.40)$ & $9.62(9.35-9.66)$ & $71(40-80)$ & $0.00(0.00-0.02)$ & $12(11-15)$ \\
\hline z5̄_PAR1_1385 & 53.273891 & -27.683685 & $5.29(5.20-5.36)$ & $9.66(9.67-9.99)$ & $30(30-71)$ & $0.16(0.12-0.22)$ & $60(42-113)$ \\
\hline z5_GSD_17901 & 53.200577 & -27.804909 & $5.33(5.23-5.43)$ & $9.91(9.57-9.97)$ & $57(30-90)$ & $0.08(0.00-0.12)$ & $34(17-48)$ \\
\hline z5_GND_34380 & 189.249146 & 62.205204 & $5.35(5.24-5.45)$ & $10.42(10.35-10.61)$ & $49(40-508)$ & $0.18(0.14-0.24)$ & $136(85-234)$ \\
\hline z5_GND_33094 & 189.266449 & 62.209164 & $5.36(5.26-5.43)$ & $9.82(9.54-9.79)$ & $49(40-80)$ & $0.10(0.02-0.10)$ & $39(16-36)$ \\
\hline z5_GNW_27194 & 189.523804 & 62.278839 & $5.40(5.30-5.50)$ & $10.14(10.01-10.30)$ & $57(40-202)$ & $0.08(0.04-0.16)$ & $50(33-105)$ \\
\hline z5_GND_39570 & 189.175720 & 62.186714 & $5.45(5.33-5.55)$ & $9.74(9.80-10.22)$ & $19(30-49)$ & $0.22(0.12-0.22)$ & $126(49-140)$ \\
\hline z5_GND_10054 & 189.169846 & 62.277298 & $5.46(5.37-5.53)$ & $9.92(9.62-9.91)$ & $57(40-90)$ & $0.08(0.00-0.14)$ & $35(16-58)$ \\
\hline z5_GND_35096 & 189.283508 & 62.203049 & $5.48(5.35-5.55)$ & $10.32(9.81-10.38)$ & $202(19-101)$ & $0.08(0.04-0.22)$ & $43(31-167)$ \\
\hline z5_GSD_4579 & 53.170231 & -27.762848 & $5.48(5.42-5.54)$ & $9.67(9.49-9.76)$ & $30(19-49)$ & $0.12(0.08-0.14)$ & $49(33-60)$ \\
\hline z5_GNW_3960 & 189.055939 & 62.129990 & $5.52(5.38-5.67)$ & $9.88(9.59-9.88)$ & $71(30-90)$ & $0.02(0.00-0.08)$ & $23(16-42)$ \\
\hline z5_GND_18617 & 189.353439 & 62.250774 & $5.53(5.46-5.63)$ & $9.68(9.56-9.91)$ & $19(19-30)$ & $0.16(0.12-0.18)$ & $95(67-122)$ \\
\hline z5_GND_24948 & 189.320312 & 62.233444 & $5.54(5.47-5.63)$ & $9.53(9.54-10.10)$ & $10(10-30)$ & $0.26(0.26-0.32)$ & $179(178-331)$ \\
\hline z5_GNW_28218 & 189.503250 & 62.273884 & $5.55(5.48-5.62)$ & $10.47(10.16-10.47)$ & $30(19-49)$ & $0.26(0.14-0.26)$ & $435(125-431)$ \\
\hline z5_GSD_4436 & 53.170811 & -27.762228 & $5.58(5.49-5.65)$ & $9.53(9.53-9.78)$ & $30(19-49)$ & $0.10(0.02-0.18)$ & $35(17-84)$ \\
\hline z5_GNW_29490 & 189.312943 & 62.344555 & $5.69(5.55-5.84)$ & $9.58(9.58-9.89)$ & $10(10-19)$ & $0.28(0.28-0.30)$ & $200(182-254)$ \\
\hline
\end{tabular}


TABLE A 3

Stellar Populations of BRight Galaxies at $z=6$

\begin{tabular}{|c|c|c|c|c|c|c|c|}
\hline ID & $\begin{array}{l}\text { Right Ascension } \\
\qquad(\mathrm{J} 2000)\end{array}$ & $\begin{array}{l}\text { Declination } \\
\text { (J2000) }\end{array}$ & Redshift & $\begin{array}{c}\log \mathrm{M}_{*} \\
\left(\mathrm{M}_{\odot}\right)\end{array}$ & $\begin{array}{l}\text { Age } \\
(\mathrm{Myr})\end{array}$ & $\mathrm{E}(\mathrm{B}-\mathrm{V})$ & $\begin{array}{c}\text { SFR } \\
\left(\mathrm{M}_{\odot} \mathrm{yr}^{-1}\right)\end{array}$ \\
\hline z6_GSD_29074 & 53.156788 & -27.839560 & $5.56(5.46-5.66)$ & $9.71(9.54-10.02)$ & $30(19-90)$ & $0.16(0.00-0.18)$ & $67(16-85)$ \\
\hline z6_GSD_17919 & 53.074165 & -27.804928 & $5.65(5.53-5.75)$ & $9.72(9.55-9.85)$ & $80(57-202)$ & $0.00(0.00-0.04)$ & $13(12-20)$ \\
\hline z6_GNW_10970 & 189.075775 & 62.172729 & $5.67(5.57-5.75)$ & $10.52(10.37-10.56)$ & $404(40-570)$ & $0.20(0.18-0.24)$ & $170(146-243)$ \\
\hline z6_GND_16399 & 189.234833 & 62.257507 & $5.67(5.58-5.76)$ & $9.66(9.44-9.75)$ & $39(19-57)$ & $0.08(0.02-0.18)$ & $27(16-87)$ \\
\hline z6_GND_16819 & 189.328232 & 62.256317 & $5.69(5.61-5.76)$ & $10.09(9.82-10.20)$ & $19(19-30)$ & $0.28(0.20-0.26)$ & $319(130-302)$ \\
\hline 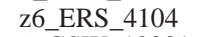 & 53.066242 & -27.689983 & $5.72(5.61-5.84)$ & $9.57(9.48-9.67)$ & $39(30-49)$ & $0.02(0.00-0.04)$ & $21(17-27)$ \\
\hline z6_GSSW_-12831 & 53.106689 & -27.930193 & $5.79(5.72-5.84)$ & $10.05(9.96-10.24)$ & $49(40-101)$ & $0.06(0.00-0.10)$ & $57(34-83)$ \\
\hline z6__GSW_6659 & 53.151745 & -27.890762 & $5.80(5.68-5.91)$ & $9.65(9.39-9.82)$ & $39(19-71)$ & $0.08(0.00-0.14)$ & $31(14-52)$ \\
\hline z6_GSD_27934 & 53.101673 & -27.836084 & $5.80(5.70-5.90)$ & $10.61(10.59-10.67)$ & $19(19-30)$ & $0.38(0.36-0.40)$ & $1066(863-1186)$ \\
\hline z6_MAIN_5871 & 53.166721 & -27.804167 & $5.80(5.76-5.83)$ & $9.90(9.64-9.91)$ & $57(40-57)$ & $0.04(0.00-0.04)$ & $34(21-34)$ \\
\hline z6_GND_28043 & 189.418427 & 62.224796 & $5.85(5.75-5.94)$ & $10.00(9.69-10.14)$ & $49(19-80)$ & $0.10(0.02-0.18)$ & $46(25-104)$ \\
\hline z6_GNW_22717 & 189.416199 & 62.333141 & $5.88(5.66-6.04)$ & $10.17(9.74-10.27)$ & $806(30-806)$ & $0.20(0.02-0.22)$ & $94(17-117)$ \\
\hline z6_GSD_23051 & 53.225368 & -27.821125 & $5.96(5.86-6.05)$ & $10.07(9.91-10.26)$ & $39(30-202)$ & $0.14(0.02-0.18)$ & $89(30-122)$ \\
\hline z6_GNW_23437 & 189.388000 & 62.301167 & $6.02(5.85-6.18)$ & $9.84(9.60-9.87)$ & $101(57-101)$ & $0.00(0.00-0.08)$ & $12(11-27)$ \\
\hline z6̄_PAR1_1068 & 53.234592 & -27.680861 & $6.13(6.05-6.23)$ & $10.10(9.90-10.16)$ & $39(40-80)$ & $0.18(0.06-0.18)$ & $109(34-103)$ \\
\hline z6_GNW_22555 & 189.301788 & 62.307808 & $6.15(6.06-6.25)$ & $9.63(9.41-9.65)$ & $57(40-57)$ & $0.02(0.00-0.02)$ & $18(12-19)$ \\
\hline z̄6_ERS_74-713 & 53.158138 & -27.702112 & $6.25(6.12-6.39)$ & $9.91(9.66-10.07)$ & $30(19-49)$ & $0.10(0.00-0.14)$ & $85(38-127)$ \\
\hline z6_GSD_21289 & 53.076241 & -27.815453 & $6.30(6.20-6.39)$ & $9.63(9.54-9.88)$ & $39(30-202)$ & $0.10(0.00-0.16)$ & $32(13-63)$ \\
\hline 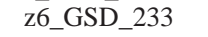 & 53.188583 & -27.733210 & $6.39(6.29-6.48)$ & 9.88 (9.42-9.93) & $30(19-49)$ & $0.20(0.04-0.20)$ & $112(23-115)$ \\
\hline
\end{tabular}

TABLE A4

Stellar Populations of BRight Galaxies at $z=7$

\begin{tabular}{|c|c|c|c|c|c|c|c|}
\hline ID & $\begin{array}{c}\text { Right Ascension } \\
(\mathrm{J} 2000)\end{array}$ & $\begin{array}{c}\text { Declination } \\
(\mathrm{J} 2000)\end{array}$ & Redshift & $\begin{array}{c}\log \mathrm{M}_{*} \\
\left(\mathrm{M}_{\odot}\right)\end{array}$ & $\begin{array}{l}\text { Age } \\
(\mathrm{Myr})\end{array}$ & $\mathrm{E}(\mathrm{B}-\mathrm{V})$ & $\begin{array}{c}\mathrm{SFR} \\
\left(\mathrm{M}_{\odot} \mathrm{yr}^{-1}\right)\end{array}$ \\
\hline z7_GSD_25074 & 53.233047 & -27.827383 & $6.66(6.47-6.79)$ & $10.09(9.87-10.15)$ & $404(49-508)$ & $0.18(0.00-0.14)$ & $82(15-55)$ \\
\hline z7_ERS_12574 & 53.094410 & -27.716846 & $6.71(6.58-6.85)$ & $9.83(9.63-10.06)$ & $49(30-202)$ & $0.08(0.00-0.18)$ & $35(16-90)$ \\
\hline z7_GNW_24443 & 189.356888 & 62.295319 & $6.72(6.65-6.81)$ & $10.02(9.80-10.05)$ & $30(30-71)$ & $0.18(0.00-0.18)$ & $155(29-160)$ \\
\hline z7_GNW_24671 & 189.361710 & 62.294373 & $6.72(6.27-7.07)$ & $9.15(8.53-9.25)$ & $19(10-40)$ & $0.10(0.00-0.10)$ & $37(11-33)$ \\
\hline z7_GSD_21368 & 53.154922 & -27.815744 & $6.75(6.62-6.97)$ & $10.14(9.89-10.34)$ & $404(30-286)$ & $0.20(0.00-0.26)$ & $82(14-142)$ \\
\hline z7_GNW_17001 & 189.032486 & 62.216415 & $6.76(6.55-6.92)$ & $10.74(10.70-10.82)$ & $404(286-570)$ & $0.06(0.02-0.12)$ & $22(14-37)$ \\
\hline z7_GSD_21172 & 53.155342 & -27.815178 & $6.84(6.76-6.96)$ & $10.19(9.95-10.20)$ & $30(19-80)$ & $0.24(0.00-0.30)$ & $231(24-396)$ \\
\hline z7_GSD_10175 & 53.210335 & -27.782211 & $7.05(6.78-7.32)$ & $9.70(9.23-9.81)$ & $19(10-71)$ & $0.22(0.00-0.24)$ & $129(15-141)$ \\
\hline z7_GND_18181 & 189.082687 & 62.252476 & $7.09(6.94-7.28)$ & $9.36(9.28-9.96)$ & $10(10-49)$ & $0.22(0.12-0.24)$ & $123(47-142)$ \\
\hline z7 GND 11402 & 189.186172 & 62.270863 & $7.16(7.00-7.34)$ & $9.69(9.22-9.76)$ & $30(10-49)$ & $0.10(0.02-0.18)$ & $51(21-114)$ \\
\hline z7_GNW_4703 & 189.094528 & 62.135540 & $7.17(7.04-7.37)$ & $9.55(9.06-9.68)$ & $39(10-49)$ & $0.02(0.00-0.10)$ & $27(22-61)$ \\
\hline z7_GNW_19939 & 189.273392 & 62.324783 & $7.25(7.03-7.75)$ & $8.79(8.64-9.29)$ & $10(10-30)$ & $0.10(0.00-0.12)$ & $34(14-42)$ \\
\hline z7_GND_42912 & 189.157883 & 62.302372 & $7.49(7.33-7.70)$ & $9.51(9.41-9.65)$ & $10(10-10)$ & $0.24(0.20-0.28)$ & $178(133-224)$ \\
\hline z7_PAR2-3098 & 53.281712 & -27.867699 & $7.66(7.44-7.82)$ & $9.82(9.56-9.90)$ & $30(19-49)$ & $0.14(0.02-0.18)$ & $69(23-111)$ \\
\hline
\end{tabular}

